\title{
Identification of dusty massive stars in star-forming dwarf irregular galaxies in the Local Group with mid-IR photometry ${ }^{\star \star \star}$
}

\author{
N. E. Britavskiy ${ }^{1,2}$, A. Z. Bonanos ${ }^{1}$, A. Mehner ${ }^{3}$, M. L. Boyer ${ }^{4}$, and K. B. W. McQuinn ${ }^{5}$ \\ 1 IAASARS, National Observatory of Athens, 15236 Penteli, Greece \\ e-mail: [britavskiy; bonanos] @astro.noa.gr \\ 2 Section of Astrophysics, Astronomy and Mechanics, Department of Physics, University of Athens, 15783 Athens, Greece \\ 3 ESO - European Organisation for Astronomical Research in the Southern Hemisphere, Santiago de Chile, Chile \\ 4 Observational Cosmology Lab, Code 665, NASA Goddard Space Flight Center, Greenbelt, MD 20771, USA \\ 5 Minnesota Institute for Astrophysics, School of Physics and Astronomy, University of Minnesota, Minneapolis, MN 55455, USA
}

Received 23 April 2015 / Accepted 5 October 2015

\begin{abstract}
Context. Increasing the statistics of spectroscopically confirmed evolved massive stars in the Local Group enables the investigation of the mass loss phenomena that occur in these stars in the late stages of their evolution.

Aims. We aim to complete the census of luminous mid-IR sources in star-forming dwarf irregular (dIrr) galaxies of the Local Group. To achieve this we employed mid-IR photometric selection criteria to identify evolved massive stars, such as red supergiants (RSGs) and luminous blue variables (LBVs), by using the fact that these types of stars have infrared excess due to dust.

Methods. The method is based on $3.6 \mu \mathrm{m}$ and $4.5 \mu \mathrm{m}$ photometry from archival Spitzer Space Telescope images of nearby galaxies. We applied our criteria to four dIrr galaxies: Pegasus, Phoenix, Sextans A, and WLM, selecting 79 point sources that we observed with the VLT/FORS2 spectrograph in multi-object spectroscopy mode.

Results. We identified 13 RSGs, of which 6 are new discoveries, as well as two new emission line stars, and one candidate yellow supergiant. Among the other observed objects we identified carbon stars, foreground giants, and background objects, such as a quasar and an early-type galaxy that contaminate our survey. We use the results of our spectroscopic survey to revise the mid-IR and optical selection criteria for identifying RSGs from photometric measurements. The optical selection criteria are more efficient in separating extragalactic RSGs from foreground giants than mid-IR selection criteria, but the mid-IR selection criteria are useful for identifying dusty stars in the Local Group. This work serves as a basis for further investigation of the newly discovered dusty massive stars and their host galaxies.
\end{abstract}

Key words. stars: massive - stars: late-type - galaxies: individual: Sex A - supergiants

\section{Introduction}

Local Group dwarf irregular galaxies (dIrrs) with high star formation rates serve as ideal laboratories for observations of all types of massive stars, as they are typically located in one compact region on the sky and are convenient for observations in multi-object spectroscopy mode. Different properties of dIrrs in the Local Group provide the opportunity to investigate the population and evolution of massive stars within the context of the different metallicities of their host galaxies. The importance of investigating massive stars, especially dusty massive stars, comes from the observed phenomenon of episodic mass loss, which complicates modeling the evolution of these objects (Smith 2014). A census of these stars in nearby galaxies is vital for our understanding of the mass-loss mechanisms and following this, the different evolutionary stages of massive stars. Thus, increasing the statistics of spectroscopically confirmed dusty massive stars, for example, red supergiants (RSGs) and luminous blue variables (LBVs), is a prerequisite for studying them in more detail.

* Based on observations made with ESO Telescopes at the La Silla Paranal Observatory under programme IDs 090.D-0009 and 091.D-0010.

$\star \star$ Appendix A is available in electronic form at http://www . aanda.org
The first obstacle in the identification of such rare stars is found in the selection process of these types of objects from photometric catalogs. A systematic search for massive stars in the Local Group became possible with the availability of highquality BVRI photometry. Massey et al. (2006, 2007c) presented optical photometry for seven star-forming dIrrs, M31 and M33, which served as a basis for further works devoted to the identification of new RSGs (Massey et al. 2009; Levesque \& Massey 2012; Drout et al. 2012; Levesque 2013), LBVs (Massey et al. 2007b; Humphreys et al. 2014; Kraus et al. 2014), and Wolf-Rayet stars (WR, Neugent \& Massey 2011) in these galaxies. In all these listed works the selection of targets was based on optical BVRI photometry, but deep optical surveys exist for a small number of dIrrs. Moreover, dusty stars appear brighter in the infrared than in optical colors because they exhibit infrared excess. Thus, infrared surveys should, in principle, provide advantages in identifying these types of stars.

We have initiated a survey that aims to provide a census of stars that have undergone episodic mass loss by using available Spitzer mid-IR photometry to select luminous mid-IR sources in a number of nearby galaxies (Khan et al. 2010, 2011, 2013; Britavskiy et al. 2014). Khan et al. aimed to identify supernova progenitors and objects similar to $\eta$ Car among the brightest targets in mid-IR colors in a number of nearby galaxies. The first attempt to systematize the selection criteria for identifying 
dusty massive stars in nearby galaxies in the mid-infrared was described in Britavskiy et al. (2014). As a result of this work, five new RSGs from eight selected candidates in the dIrr galaxies Sextans A and IC 1613 were spectroscopically confirmed, which provides some evidence for the success of mid-IR selection criteria for dusty massive stars.

In this paper we continue our work of identifying dusty massive stars in the dIrr galaxies of the Local Group. We base our selection approach for interpreting luminous, massive, resolved stellar populations in nearby galaxies at infrared wavelengths on the roadmap presented by Bonanos et al. (2009, 2010). RSGs, supergiant $\mathrm{B}[\mathrm{e}](\mathrm{sgB}[\mathrm{e}])$ stars, and LBVs are among the brightest infrared sources because of their intrinsic brightness and because of dust, and they occupy distinct regions in all CMDs. The paper is organized as follows: in Sect. 2 we briefly review the procedure for selecting sources and describe the observations and data reduction of the obtained spectra. In Sect. 3, we discuss the spectral classification process. Section 4 presents our results, grouped by object type, in subsections. In Sect. 5, we discuss our results and evaluate the selection criteria. Section 6 closes the paper with a summary.

\section{Target selection and observations}

\subsection{Target selection}

Following Britavskiy et al. (2014), we selected evolved massive star candidates from published Spitzer/IRAC photometry (Boyer et al. 2009) of four nearby irregular dwarf galaxies with relatively high star formation rates $\left(\gtrsim 0.003 M_{\odot} \mathrm{yr}^{-1}\right)$ : Pegasus, Phoenix, Sextans A, and WLM. The selection of galaxies was made by taking into account the occurrence of recent star-formation episodes (Mateo 1998). All program galaxies have a few recent star formation populations (Tolstoy 1999; Hodge et al. 1999), except for the low-mass Phoenix galaxy, which has one central, young (100 Myr) stellar population zone (Martínez-Delgado et al. 1999).

Luminous blue variables, sgB[e]s, and RSG stars are among the brightest stars at $3.6 \mu \mathrm{m}$ and are concentrated on the colormagnitude diagrams (CMDs) at $M_{3.6 \mu \mathrm{m}}<-9 \mathrm{mag}$. OB stars are located along a vertical line at [3.6]-[4.5] $\sim 0$ mag. RSGs also fall on this line, but are separated by their extreme brightness, therefore RSG candidates were selected as objects with colors [3.6]-[4.5] $<0$ and $M_{3.6 \mu \mathrm{m}}<-9$ mag. LBVs, $\mathrm{sgB}[\mathrm{e}] \mathrm{s}$, and WR stars have redder colors than OB stars. To find new LBVs and $\mathrm{sgB}[\mathrm{e}]$ we selected all sources in Boyer et al. (2009) with $M_{3.6 \mu \mathrm{m}} \leq-8 \mathrm{mag}$ and [3.6]-[4.5] $>0.15 \mathrm{mag}$. Additional bright IR sources at smaller [3.6]-[4.5] color were observed when it was possible to add slits on the FORS2 spectroscopic masks (multi-object MXU mode) without compromising the main targets. Following Bonanos et al. (2009, 2010), the selection criteria presented above include LBVs, $\operatorname{sgB}[\mathrm{e}] \mathrm{s}$, WR stars, and RSGs, but exclude normal OB stars. For the Phoenix galaxy, we selected candidates with absolute magnitude $M_{3.6 \mu \mathrm{m}}<-6 \mathrm{mag}$ because all targets were relatively faint. In this way, we filled the remaining free space on the multi-object mask (MXU).

The selected candidates were cross-correlated with existing optical and near-infrared catalogs to eliminate known foreground stars, H II regions, galaxies, and radio sources. In the end, 43 high-priority targets were selected, but to fill the free space on the MXU masks, we added 36 more targets. These additional targets were selected among the brightest sources in the [3.6] band, but quite often they were fainter than the magnitude cutoff criteria $\left(M_{3.6} \mu \mathrm{m} \leq-9 \mathrm{mag}\right)$, and they did not always satisfy our selection criteria in terms of the [3.6]-[4.5] color. Moreover, 10 of these additional targets have been reported as candidate carbon stars based on their photometry (Battinelli \& Demers 2000, 2004; Menzies et al. 2008). In total, 79 stars were observed in four dIrr galaxies: 19 in Pegasus, 14 in Phoenix, 15 in Sextans A, and 31 in WLM.

After the observations were carried out, new mid-IR photometry became available from the DUSTiNGS survey (DUST in Nearby Galaxies with Spitzer Boyer et al. 2015b). This survey includes 3.6 and $4.5 \mu \mathrm{m}$ imaging of 50 nearby dwarf galaxies within $1.5 \mathrm{Mpc}$, which are observed with the aim to identify dust-producing asymptotic giant branch (AGB) stars. Deep images were obtained with the IRAC camera onboard the Spitzer telescope during the post-cryogen phase on two epochs, with an average difference of 180 days. Since the new photometry from DUSTiNGS has better accuracy, we used the new [3.6] and [4.5] values (Epoch 1) for our analysis, which vary from the old ones by 0.05-0.2 mag in both the [3.6] and [4.5] bands, even though the selection process was based on the published photometry from Boyer et al. (2009). Given that the spatial resolution of Spitzer/IRAC photometry at [3.6] and [4.5] bands is 2", which corresponds to $\approx 10 \mathrm{pc}$ at a distance $1 \mathrm{Mpc}$, it is unlikely to have two bright mid-IR sources in this area.

\subsection{Observations and data reduction}

The sample of selected targets was observed with the FORS2 spectrograph at ESO's Very Large Telescope during two observing runs (program IDs 090.D-0009 and 091.D-0010). The observations were carried out in multi-object spectroscopy mode, which maximizes the number of selected targets in one observational set. For each galaxy the observations were performed in one MXU field, except for observations of Sextans A and WLM, where observations were performed in two MXU fields. In Sextans A, one MXU field was observed twice, while for WLM the observations were performed in two different observational fields. The journal of observations and properties of observed galaxies, that is, the time of observation (MJD), exposure time, distance modulus, systematic radial velocities and metallicities, is given in Table 1.

The data reduction of the observed targets was performed with the FORS pipeline recipes version 4.9.23 under ESO Reflex workflows version 2.6 (Freudling et al. 2013). With the help of this pipeline, 1D spectra were automatically extracted from the raw image. The reduction process included standard procedures such as bias subtraction, flat field division, background subtraction, and wavelength calibration. For each field, observation spectra were obtained four times and combined using the IRAF $^{1}$ scombine routine. The final average extracted 1D science spectra were normalized using the continuum procedure. All spectra were flux calibrated using moveable slit (MOS) mode observations of the flux standard stars. The spectra have an average signal-to-noise ratio $(\mathrm{S} / \mathrm{N})$ of $\approx 20$ and a spectral range from $4300 \AA$ to $9000 \AA$. We did not achieve this wavelength range for all spectra; some of them have a shorter range, which made spectroscopic analysis difficult. The resolving power varies from $R \approx 400$ at $5000 \AA$ to $R \approx 680$ at $8600 \AA$.

1 IRAF is distributed by the National Optical Astronomy Observatory, which is operated by the Association of Universities for Research in Astronomy (AURA) under cooperative agreement with the National Science Foundation. 
Table 1. Journal of observations and target galaxy properties.

\begin{tabular}{lccccc}
\hline \hline ID & $\begin{array}{c}\text { MJD } \\
(\mathrm{JD}-2400000.5)\end{array}$ & $\begin{array}{c}\text { Exp. } \\
(\mathrm{s})\end{array}$ & $\begin{array}{c}\text { Distance modulus } \\
(\mathrm{mag})\end{array}$ & $\begin{array}{c}\text { Radial velocity } \\
\left(\mathrm{km} \mathrm{s}^{-1}\right)\end{array}$ & $\begin{array}{c}{[\mathrm{Fe} / \mathrm{H}]} \\
(\mathrm{dex})\end{array}$ \\
\hline Pegasus & 56487.34940 & $4 \times 660$ & $24.82 \pm 0.07$ & $-183.3 \pm 5$ & -1.40 \\
Phoenix & 56279.13559 & $4 \times 660$ & $23.09 \pm 0.10$ & $-13 \pm 9,-52 \pm 6$ & -1.37 \\
Sextans A (Set 1) & 56360.08635 & $4 \times 660$ & $25.78 \pm 0.08$ & $+324 \pm 2$ & -1.85 \\
Sextans A (Set 2) & 56423.08622 & $4 \times 660$ & - & - & - \\
WLM (Objects 1-18) & 56279.08819 & $4 \times 660$ & $24.85 \pm 0.08$ & $-130 \pm 1$ & -1.27 \\
WLM (Objects 19-31) & 56280.10188 & $4 \times 660$ & - & - & - \\
\hline
\end{tabular}

Notes. The distance moduli, systemic radial velocities and metallicities are taken from McConnachie (2012). The second systematic RV value for Phoenix is from Gallart et al. (2001).

\section{Spectral classification process}

The spectral analysis consists of the following steps: (i) radial velocity (RV) determination; (ii) spectral type determination; and (iii) luminosity class classification. This is the same algorithm as was used to identify RSGs in Britavskiy et al. (2014).

We determined the spectral type using the ESO UVES Paranal Observatory Project (POP) library of high-resolution spectra, which covers a wide range in luminosity and colors (Bagnulo et al. 2003). We decreased the resolution of the templates from $R \approx 70000$ to a resolution of $R=440$ to match our spectra. This value of the resolution was chosen as an average resolution of the obtained spectra as claimed in the FORS2 spectrograph tutorial. At this resolution it is quite difficult to distinguish the lines that are indicators of spectral type - instead of lines, we only have blends. Nevertheless, the comparison with templates provides the opportunity to investigate the behavior of the TiO bands, which are very strong for $\mathrm{K}$ and $\mathrm{M}$ spectral types. By using 17 spectral templates with a broad range of spectral types (from F2 to M6), we provide a spectral determination accurate to the level of one late or early spectral subtype.

The determination of luminosity class was based on the investigation of the $\mathrm{Ca}$ II line profiles by comparing the strengths of these gravity-sensitive features with giant and supergiant template spectra. We used the sample of confirmed RSGs and foreground giants in Sextans A from Britavskiy et al. (2014) as templates. We also used the Ca II triplet for measuring RVs; we cross-correlated spectral templates from the NASA Infrared Telescope Facility spectral library for cool stars (Rayner et al. 2009) against the Ca II region ( $\lambda \lambda 8380-8800 \AA)$ of our program stars using the IRAF task fxcor. When the Ca II region was absent from the spectra, the radial velocity analysis was not performed. We used different libraries to determine the spectral type and luminosity because the Ca II triplet region is not included in the ESO UVES POP library.

For a quantitative analysis of the Ca II triplet profiles we used the empirical calibration of the near-IR index CaT (defined by Cenarro et al. 2001), which measures the Ca II triplet strength. We calculated indices and errors for each star spectrum where the Ca II triplet existed by using the indexf package (Cardiel 2010). For foreground giants this index $(\mathrm{CaT} \simeq 3-7)$ is typically half that of supergiants $(\mathrm{CaT}>10)$ and agrees with the empirical fitting-function library (Cenarro et al. 2002) for stars that have the same spectral type and metallicity. The CaT index measurements confirm the supergiant nature for all stars that have been identified as RSGs from the comparison with template spectra described above.

The resulting spectral type classification of targets and CaT index, when available, is shown in Tables A.1-A.4. These tables list a running ID number and ID from the DUSTiNGS of all observed targets, their coordinates, radial velocities (RV), optical magnitude and colors (from Massey et al. 2007c), absolute [3.6] mag $\left(M_{[3.6]}\right.$, computed using the distance in Table 1), [3.6]-[4.5] colors, the final spectral classification and notes. When it was not possible to determine the luminosity class, for example, when the spectrum did not include the Ca II triplet region, we provide only the spectral type. The column "Notes" lists other spectroscopic information from the literature. The label "Unclassified" was used for the targets with low-quality spectra or spectra with high noise that did not allow a spectral type determination.

\section{Results}

Figures 1-4 present the color-magnitude diagrams (CMD) and spatial distribution of our targets in the four program galaxies, Phoenix, Pegasus, Sextans A, and WLM, respectively. In the $M_{\text {[3.6] }}$ vs. [3.6]-[4.5] CMDs we kept the same formalism of object classification (labels) as we used in Tables A.1-A.4. In each CMD we highlight the regions that satisfied our photometric selection criteria in light gray. The region in the left part corresponds to the area where RSGs are expected ([3.6]-[4.5] $<0$ ), the region to the right corresponds to the $\mathrm{LBV}$ and $\mathrm{sgB}[\mathrm{e}]$ stars ([3.6]-[4.5] > 0.15). The value of the absolute magnitude cutoff varies from galaxy to galaxy. For example, the cutoff was decreased to $M_{3.6}<-6 \mathrm{mag}$ in Phoenix, since all targets in this galaxy are relatively faint in comparison with the other galaxies. In WLM, the cutoff was changed to $M_{3.6}<-8$ mag to select more targets in the LBV region because two MXU masks were available per field. All unclassified objects are faint and have large error bars in the [3.6]-[4.5] color, in comparison with the objects for which spectral classification was performed successfully. In some cases the absolute magnitude of the targets is below the magnitude selection criteria cutoff $M_{3.6}<-9$ mag. This discrepancy arises because the selection was based on older photometry, while the CMDs plot the more accurate DUSTiNGS photometry.

The analysis of the spatial distribution of observed targets shows that the majority of the foreground and background objects are located outside the main body of dIrrs. Thus, we can conclude that an additional check of the position of candidates with respect to the host galaxy body will help avoid contamination of foreground or background objects.

Among the observed targets we select several groups of dusty massive stars that are described in the subsections below.

\subsection{Red and yellow supergiants}

In Fig. 5 we show spectra of all 13 identified RSGs from all galaxies and one candidate yellow supergiant (Sex A 4), ordered 

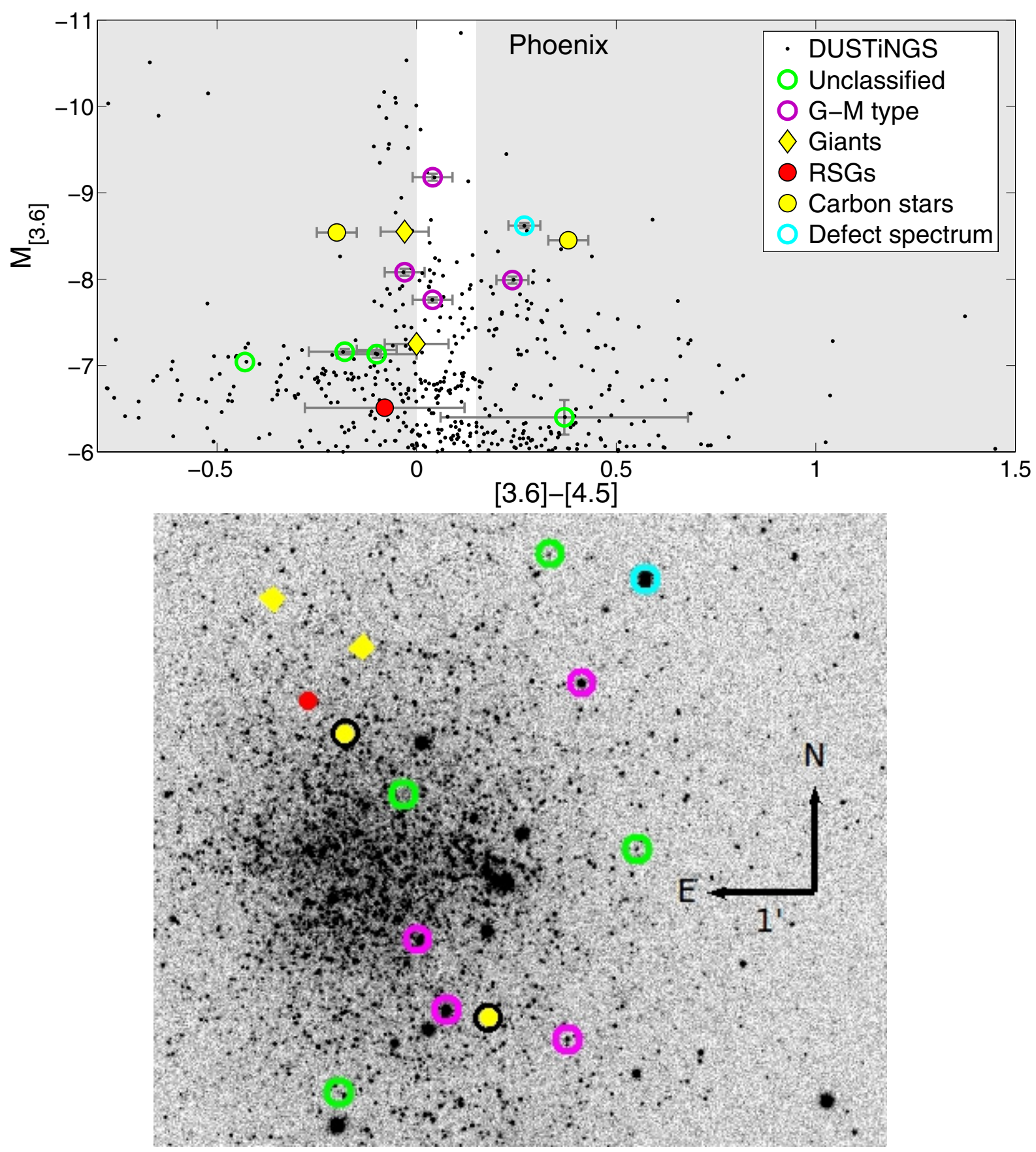

Fig. 1. Top panel: $M_{[3.6]}$ vs. [3.6]-[4.5] CMD for the Phoenix dIrr galaxy. The stars that we observed are indicated by different symbols according to their classification, given in Table A.1 as explained in the legend. For each target on the CMD we indicate the error bars for the colors and magnitudes by gray lines. The regions that satisfy our selection criteria are highlighted in light gray (the left region corresponds to the RSGs area, the right region corresponds to the LBVs and $\mathrm{sgB}[\mathrm{e}]$ area, see text for more details). Bottom panel: the spatial distribution of the observed targets, superposed on $V$-band images of the Phoenix galaxy (Massey et al. 2007c).

by spectral type. Most of the RSGs have K spectral type, except for two M-type RSGs. The low resolution of our spectral data does not allow us to resolve individual lines, which are indicators of luminosity class or spectral type. The main features that are visible is the $\mathrm{Ca}$ II triplet and dominant $\mathrm{TiO}$ bands in the optical part of RSGs spectra (see Fig. 5). Thus, only the molecular blends are visible in the spectra. It was possible to resolve some additional lines in the early-type spectrum of Sex A 4. An example of more detailed spectral type and luminosity class identification for red and yellow supergiants from optical wavelength range spectra can be found in Ginestet et al. (1994), Negueruela et al. (2012), Drout et al. (2012), and Britavskiy et al. (2014).
The spectrum of Sex A 4 has strong hydrogen lines from Paschen series in the Ca II triplet region, but the main feature of this type, the O I $7774 \AA$ triplet, is absent. The strong absorption lines of $\mathrm{H} \alpha$ and $\mathrm{H} \beta$ in the Sex A 4 spectrum, suggest a G or F spectral type for this target.

In total, we identify 13 RSGs, 6 of which are new discoveries; 4 were previously published in the independent study of Levesque \& Massey (2012), which used optical selection criteria, and 3 RSGs were previously identified by Britavskiy et al. (2014). By applying our mid-IR selection criteria, we selected these targets independently of Levesque \& Massey (2012). Given two separate spectroscopic observations of these four 
N. E. Britavskiy et al.: Identification of dusty massive stars in nearby galaxies
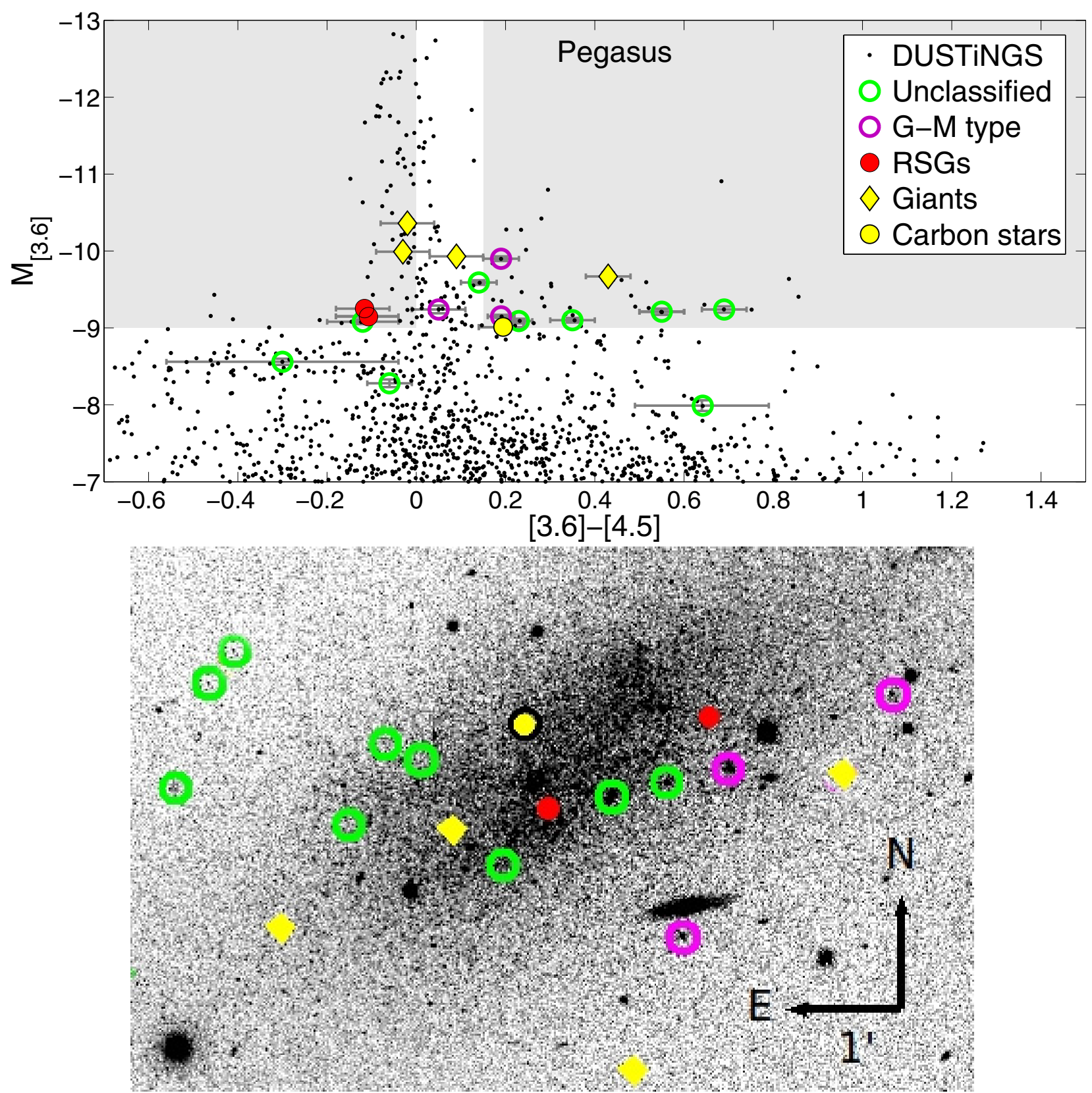

Fig. 2. Same as Fig. 1, but for the Pegasus dIrr galaxy. The stars that we observed are indicated by different symbols according to their classification, given in Table A.2.

objects, carried out four years apart, we can compare their spectral type and check for spectral variability (Massey et al. 2007a; Levesque 2010). Our spectroscopic analysis (see Table A.4 and Fig. 6) does not show any significant difference in spectral type.

The positions of all spectroscopically confirmed RSGs in the CMD are predicted by our selection criteria, nevertheless, as we can see in Fig. 4, one RSG in WLM (object J000158.14152332.2 in Levesque \& Massey 2012) is located in the red part ([3.6]-[4.5] > 0.15) of the CMD. Britavskiy et al. (2014) also identified one RSG ("IC 1613 1") with an unusual color, [3.6]-[4.5] $=0.66 \mathrm{mag}$. This color behavior might be explained by a lack of molecular $\mathrm{CO}$ and $\mathrm{SiO}$ features in the [4.5] band (Verhoelst et al. 2009). Alternatively, the explanation might be the photometric variability of these RSGs (see discussion in Britavskiy et al. 2014).

The low radial velocity values of RSGs in WLM, namely WLM 29, WLM 30, WLM 31, which are different from other objects from this galaxy (e.g., WLM 14 and WLM 17, see Table A.4), as well as their spatial distribution (these three RSGs are located close to each other, to the south with respect to the main body of WLM, see Fig. 4) suggest a high rotation velocity for this galaxy. The extragalactic origin of the observed targets was determined by considering the radial velocities together with the spectral and luminosity class determination, as radial velocities alone do not always distinguish foreground objects from extragalactic ones.

\subsubsection{Foreground giants}

We identified eight K-M spectral type giants that are foreground stars in the Milky Way: two giants in Phoenix, four giants in Pegasus, and one giant in the Sextans A and WLM dIrr galaxies. As a result of their late spectral types, they have the same magnitude and position in the CMD diagrams as RSGs. In the context 

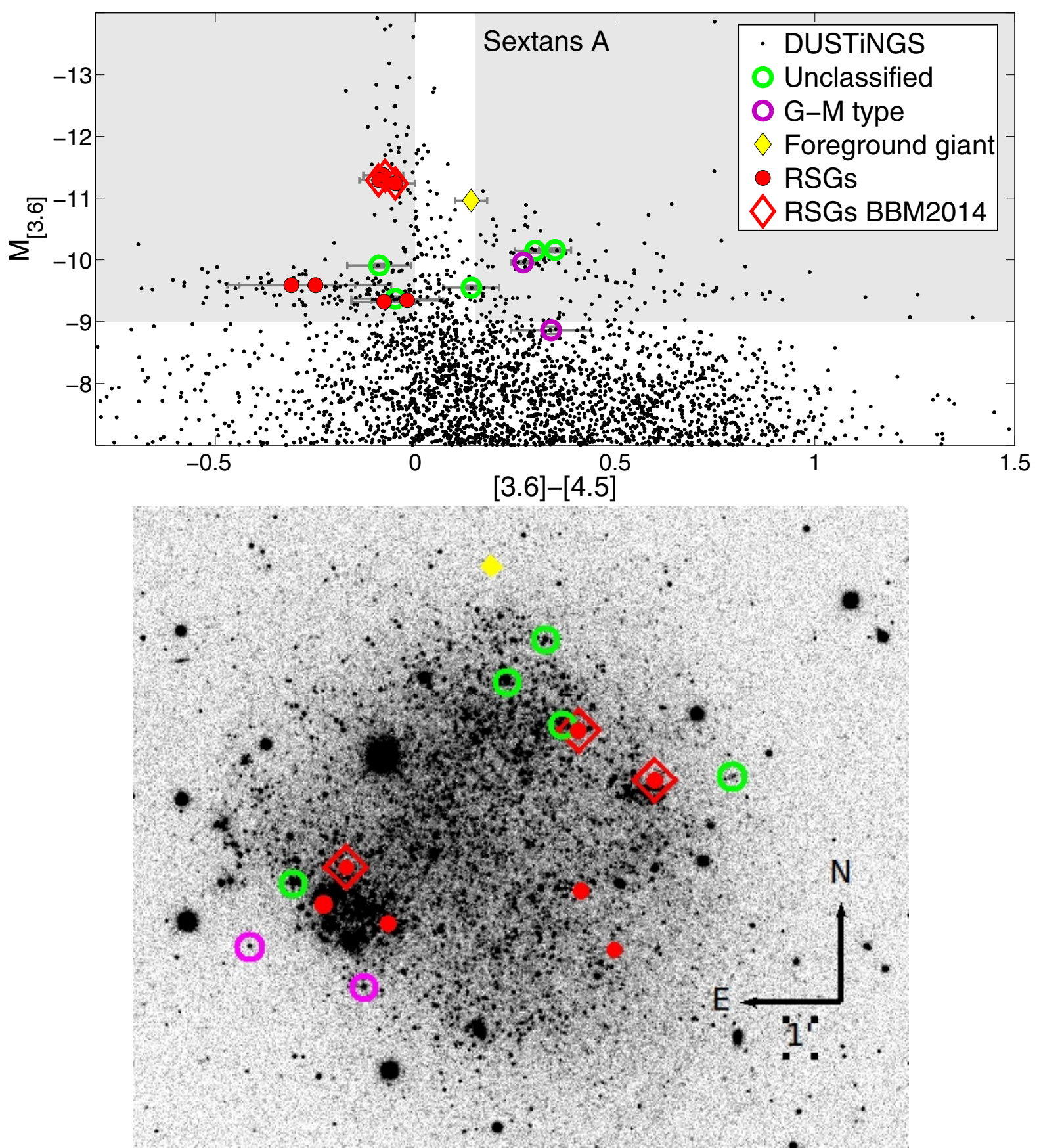

Fig. 3. Same as Fig. 1, but for the Sextans A dIrr galaxy. The stars that we observed are indicated by different symbols according to their classification, given in Table A.3. All previously known RSGs are marked by open red diamonds.

of the mid-IR selection criteria, giants are hardly distinguishable from RSGs, as discussed further in Sect. 5. Their classification as giants was based on the relatively weak Ca II triplet and lower radial velocities compared with RSGs in the same host galaxies.

\subsection{Emission line stars}

We discovered two rare emission line objects in WLM, WLM 17 and WLM 23, whose spectra are presented in Fig. 7. A list of identified emission lines for each spectrum is presented in Table 2. Line identification was performed by using the spectral atlas of $\eta$ Car (Zethson et al. 2012) and the spectral atlas of earlytype hypergiants, including LBVs and $\mathrm{sgB}[\mathrm{e}]$ stars (Chentsov et al. 2003). The spectral resolution does not allow us to perform an accurate spectroscopic classification of these targets or to investigate the hydrogen line profiles, but we can provide some information about the nature of these sources.

In addition to strong hydrogen lines in emission, object WLM 17 has some forbidden lines of high excitation potential, such as [O II] and [Ar III], which indicate a high temperature of the stars, about $T_{\text {eff }} \approx 30000 \mathrm{~K}$. The nebular lines [N II] $6583 \AA$ and [S II] $6717 \AA$ and $6731 \AA$ indicate that this star is surrounded by an envelope. The position of this object in the $\mathrm{H} \alpha$ image of WLM (Massey et al. 2007c) shows that this object lies inside an HII region. Furthermore, according to Hodge \& Miller (1995), WLM 17 is located near an identified HII region, and spectroscopic features such as [O III] 5007, He I 5876, N II 6984, He I 6678, and [Ar III] $7135 \AA$ originate from the 
N. E. Britavskiy et al.: Identification of dusty massive stars in nearby galaxies

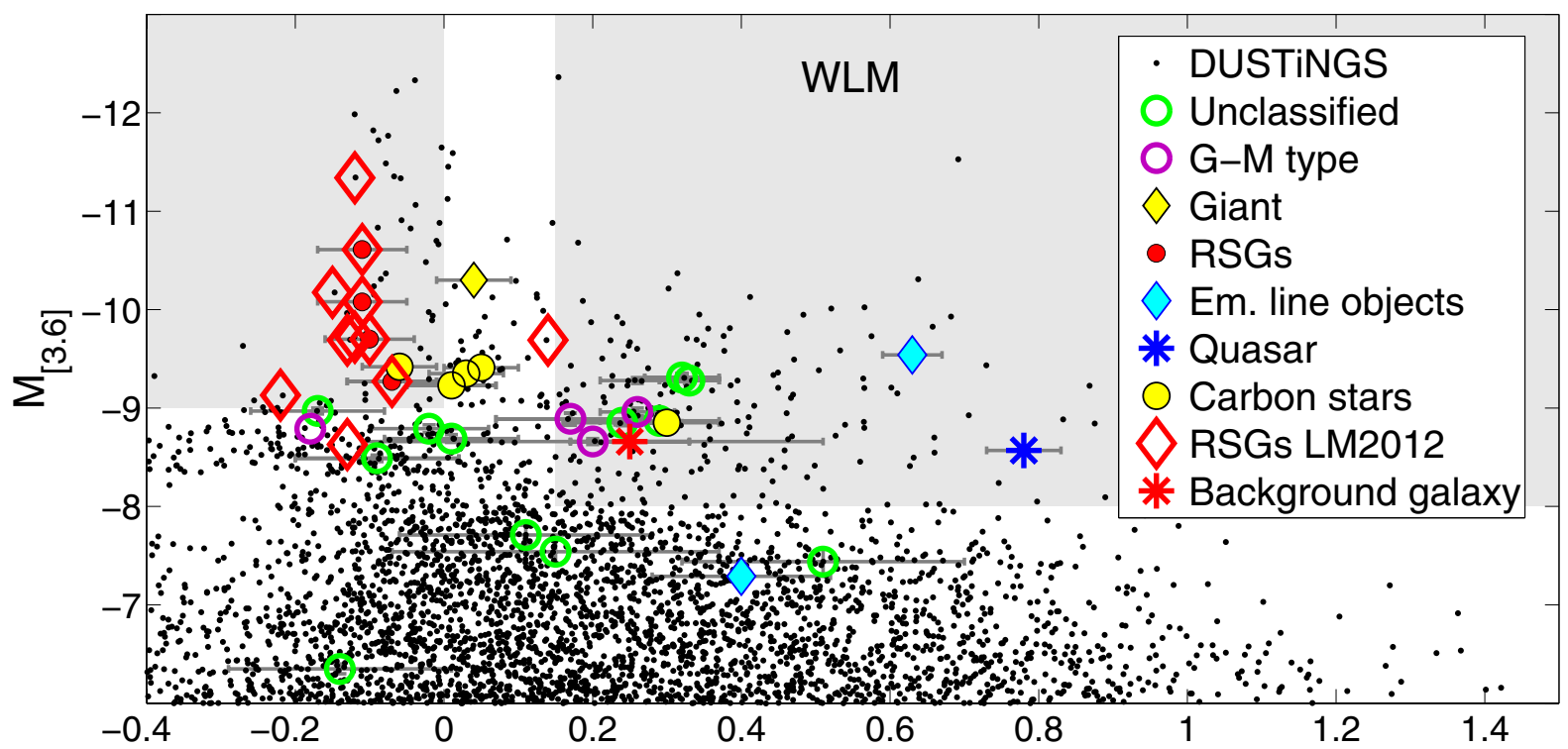

[3.6]-[4.5]

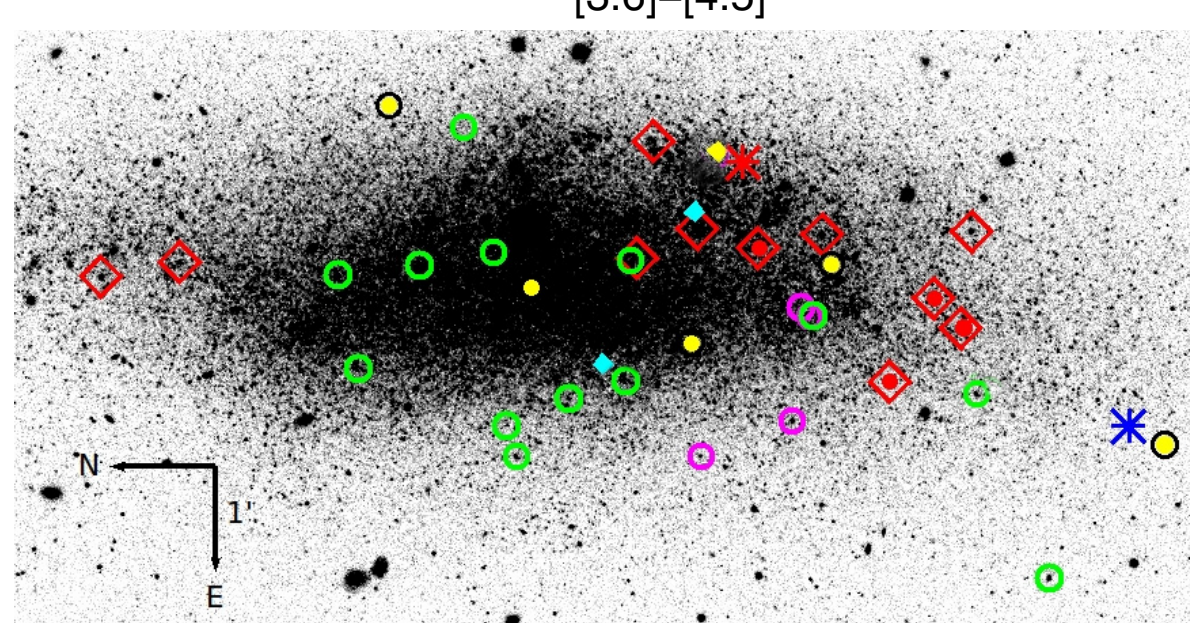

Fig. 4. Same as Fig. 1, but for the WLM dIrr galaxy. The stars that we observed are indicated by different symbols according to their classification, given in Table A.4. All previously known RSGs are marked by open red diamonds, emission line objects by blue filled diamonds, and background objects by differently colored stars.

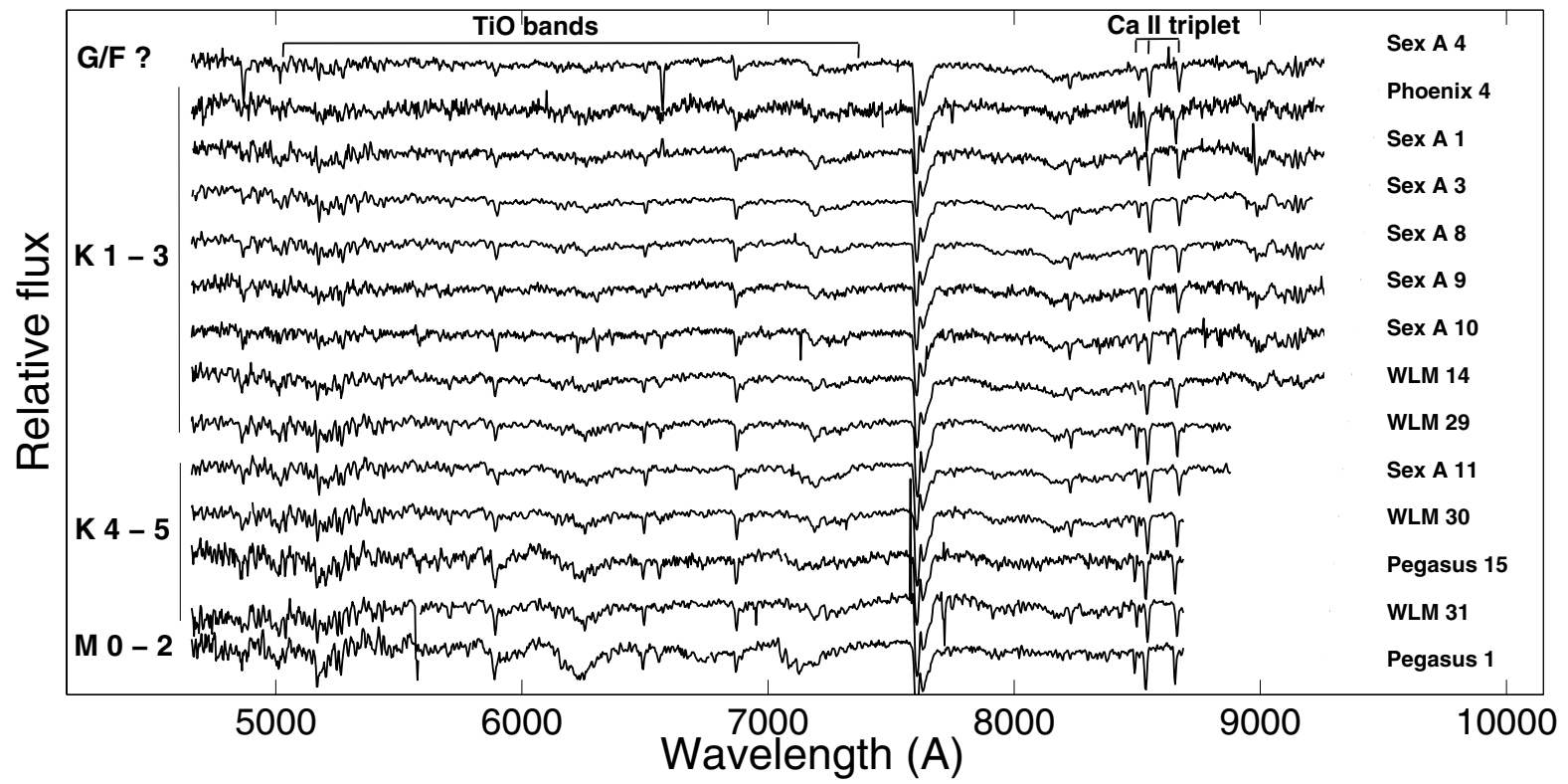

Fig. 5. Spectra of our 13 RSGs and one candidate yellow supergiant (Sex A 4) in the optical range, ordered by spectral type, labeled on left. Names are given on right. The region where $\mathrm{TiO}$ bands dominate, along with the $\mathrm{Ca}$ II triplet are marked. 
Table 2. Identified emission lines in WLM 17 and WLM 23.

\begin{tabular}{|c|c|c|c|}
\hline \multicolumn{2}{|c|}{ WLM 17} & \multicolumn{2}{|c|}{ WLM 23} \\
\hline Ion & $\begin{array}{l}\text { Wavelength } \\
\text { (A) }\end{array}$ & Ion & 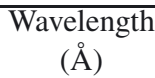 \\
\hline$\overline{\mathrm{H} \gamma}$ & 4340 & $\mathrm{H} \gamma$ & 4340 \\
\hline $\mathrm{H} \beta$ & 4861 & - & 4501 \\
\hline$[\mathrm{O} \mathrm{II}]+[\mathrm{Fe} \mathrm{II}]$ or & 4957 & $\mathrm{H} \beta$ & 4861 \\
\hline [O III] ? & 4960 & $\mathrm{Fe}$ II & 4925 \\
\hline$[\mathrm{Fe}$ II] or [O III] & 5008 & {$[\mathrm{O} \mathrm{II}]+[\mathrm{Fe} \mathrm{III}]$} & 4957 \\
\hline$[\mathrm{Fe}$ II $]$ & 5045 & {$[\mathrm{Fe} \mathrm{II}]$} & 5008 \\
\hline S II & 5453 & $\mathrm{Fe}$ II & 5020 \\
\hline $\mathrm{Fe}$ II & 5582 & $\mathrm{Fe}$ II & 5170 \\
\hline$[\mathrm{Fe} \mathrm{II]}$ & 5655 & $\mathrm{Fe}$ II & 5236 \\
\hline$[\mathrm{Fe} \mathrm{II}]$ & 5871 & Fe II & 5277 \\
\hline He I & 5876 & Fe II & 5318 \\
\hline $\mathrm{Fe}$ II & 6249 & $\mathrm{Fe}$ II & 5536 \\
\hline $\mathrm{H} \alpha$ & 6564 & $\mathrm{Ne} I ?$ & 6165 \\
\hline$[\mathrm{N}$ III] & 6584 & Fe II & 6249 \\
\hline $\mathrm{He} \mathrm{I}$ & 6678 & {$[\mathrm{O} \mathrm{I}]$} & 6300 \\
\hline [S II] & 6717 & $\mathrm{Fe}$ II & 6458 \\
\hline [S II] & 6731 & $\mathrm{Fe}$ II & 6518 \\
\hline [Ar III] & 7135 & $\mathrm{H} \alpha$ & 6564 \\
\hline $\mathrm{He} \mathrm{I}$ & 7283 & O I ? & 7774 \\
\hline O II & 7321 & O I & 8446 \\
\hline- & 7723 & H I P17 & 8467 \\
\hline S II & 7852 & Ca II & 8498 \\
\hline- & 8565 & Ca II & 8542 \\
\hline H I P14 & 8598 & H I P14 & 8600 \\
\hline Ca II & 8662 & Ca II & 8662 \\
\hline $\mathrm{Fe}$ II & 8674 & H I P12 & 8750 \\
\hline $\mathrm{Cr}$ II & 8832 & $\mathrm{Cr}$ II & 8830 \\
\hline [S III] ? & 9071 & H I P11 & 8863 \\
\hline $\mathrm{Fe}$ II & 9073 & $\mathrm{Fe}$ II & 8929 \\
\hline & & H I P10 & 9014 \\
\hline & & H I P9 & 9229 \\
\hline
\end{tabular}

Notes. Controversial line identifications are indicated by a question mark.

HII region. We conclude that this object is an evolved, hot, and young massive star near an HII region.

Object WLM 23 has strong hydrogen emission, but the absence of He lines and forbidden Fe lines indicates a lower temperature for this star, $T_{\text {eff }}<20000 \mathrm{~K}$. The spectrum exhibits both the Ca II triplet and Paschen hydrogen series in emission. Furthermore, dominant Fe II emission lines in the spectrum suggest that this object is an iron star (see Sect. 2.2 in Humphreys et al. 2014). These two objects were selected as candidate LBVs or sgB[e] stars ([3.6]-[4.6] > $0.15 \mathrm{mag}$, see Fig. 4). He emission lines and forbidden lines of $\mathrm{Fe}$ are expected to exist in these types of stars, but we did not detect them. By taking into account the abundance of Fe II emission lines and the high absolute magnitude $\left(M_{[3.6]}=-9.54 \mathrm{mag}\right)$, we suggest that object WLM 23 is a hypergiant that belongs to the class of Fe II emission stars, which were defined by Clark et al. (2012). This object is the first identified Fe II emission line star in the dIrr galaxies of the Local Group.

\subsection{Carbon stars}

Of the bright sources in mid-IR colors, carbon stars also satisfy our selection criteria. Although some candidates were listed in the literature as candidates for carbon stars (Battinelli \& Demers 2000, 2004; Menzies et al. 2008), we still observed them to fill the free space on MXU masks. In total we observed ten photometric candidates, eight of which are spectroscopically confirmed as carbon stars in this work and are presented in Fig. 8. For the other two carbon star candidates it was not possible to determine the spectral type. The typical spectroscopic molecular features of carbon stars, CN at 6900-7500 $\AA$ and 7900-8400 $\AA$, are detected in all spectra. For a more precise determination of the spectral type we used template spectra of various carbon stars from the SDSS-III archive. From the behavior of the dominant C2 Swan bands in the $4600 \sim 5600 \AA$ region, we conclude that these objects are all late-type carbon stars, presumably C-N 6-7 series, which, according to the spectral classification in Keenan (1993), are equivalent to early M-type stars. For a diagnostic of the luminosity class we used the ratio of the C2 $5165 \AA$ band to Mg I $5186 \AA$, which suggests that these objects have a low surface gravity, $\log g<1$, corresponding to giants. Some of the identified carbon stars are listed as newly identified variable dusty massive AGB stars, according to Boyer et al. (2015a). These targets are indicated with "VAR" in Tables A.1-A.4.

\subsection{Background objects}

We identified two objects among the WLM targets that belong to the background: a quasar (WLM 26) and a background galaxy (WLM 15). By using template spectra from the SDSS-III archive (Data Release 9, Ahn et al. 2012), we determined the redshift of the quasar to be $z=0.62$ based on the $\mathrm{H} \beta$ and [O III] lines, and the redshift of the galaxy is estimated to be $z=0.39$ based on the Ca II $3968 \AA$ line. The spectra of these two objects are presented in Fig. 9. The absence of emission lines in the spectrum of the galaxy implies that it is an early-type galaxy, according to the SDSS-III classification. These objects might be applied as probes of the interstellar medium in WLM (an example of using radio pulsars for this purpose is presented in Kondratiev et al. 2013). Background quasars can also be used to determine the proper motion of dIrr galaxies in the Local Group, as done in the Magellanic Clouds (Kozłowski et al. 2013).

\section{Discussion}

Since the main goal of this paper was to identify as many dusty massive stars as possible, the critical question is the efficiency of the selection criteria. The discovery of new RSGs and emission line stars provides an opportunity to test the success rate of our criteria. In Table 3 we present the classification of targets in each program galaxy. We provide the following seven categories: (i) "Unclassified" - targets mainly with low S/N spectra, for which we were not able to provide a classification; (ii) "Spectral type only" - targets for which only the spectral type identification was performed; (iii) "Giants" - foreground giants; (iv) "RSGs"; (v) "Em. line objects" - emission line objects; (vi) "Background objects" - quasar (WLM 26) and background galaxy (WLM 15); (vii) "Carbon stars". The number of targets satisfying our selection criteria are highlighted in bold font, while the number of targets that were randomly selected to fill the free space on the MXU masks are listed in parentheses. The percentage of the selected targets belonging to each of these categories is listed in the last line of the table. The percentage of evolved massive targets is $30 \%$ for RSGs and $5 \%$ for emission line objects. The accuracy of the mid-IR photometry that was used for the selection process is likely responsible for the low percentages. In addition, we expect that evolved massive stars exist among the "unclassified" and "spectral type only" targets, 

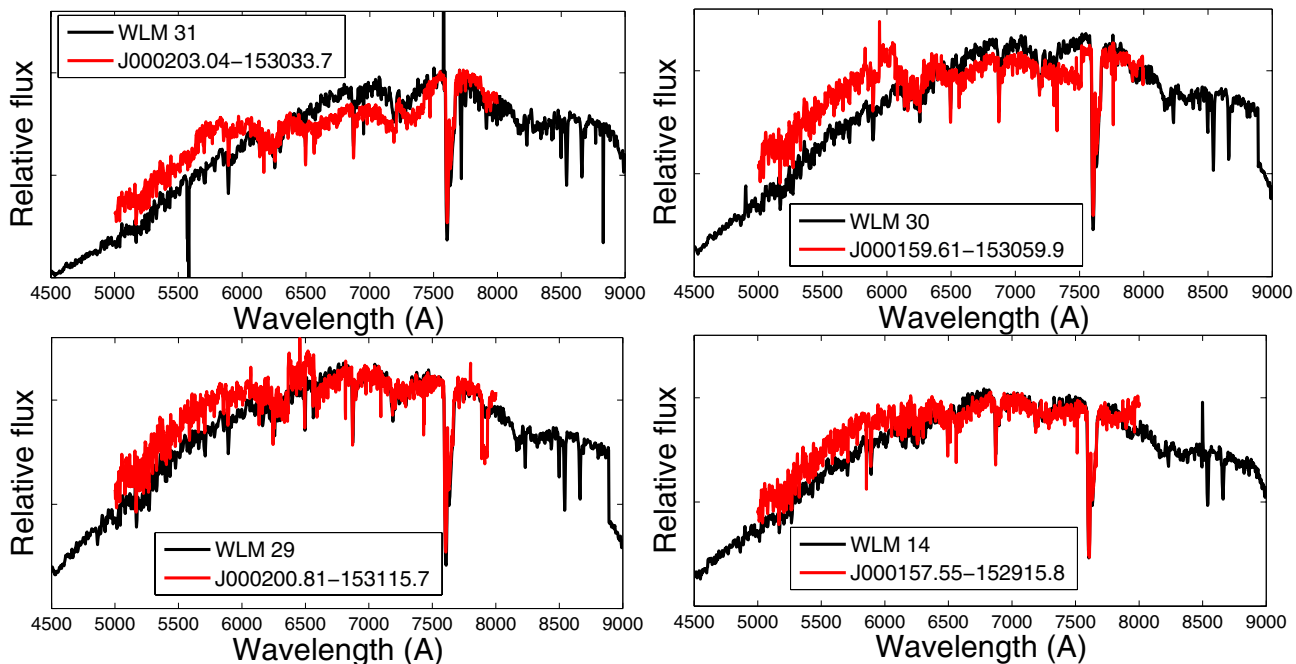

Fig. 6. Comparison of 4 FORS2 spectra (black) out of 7 previously known RSGs with the spectra of Levesque \& Massey (2012) (red).

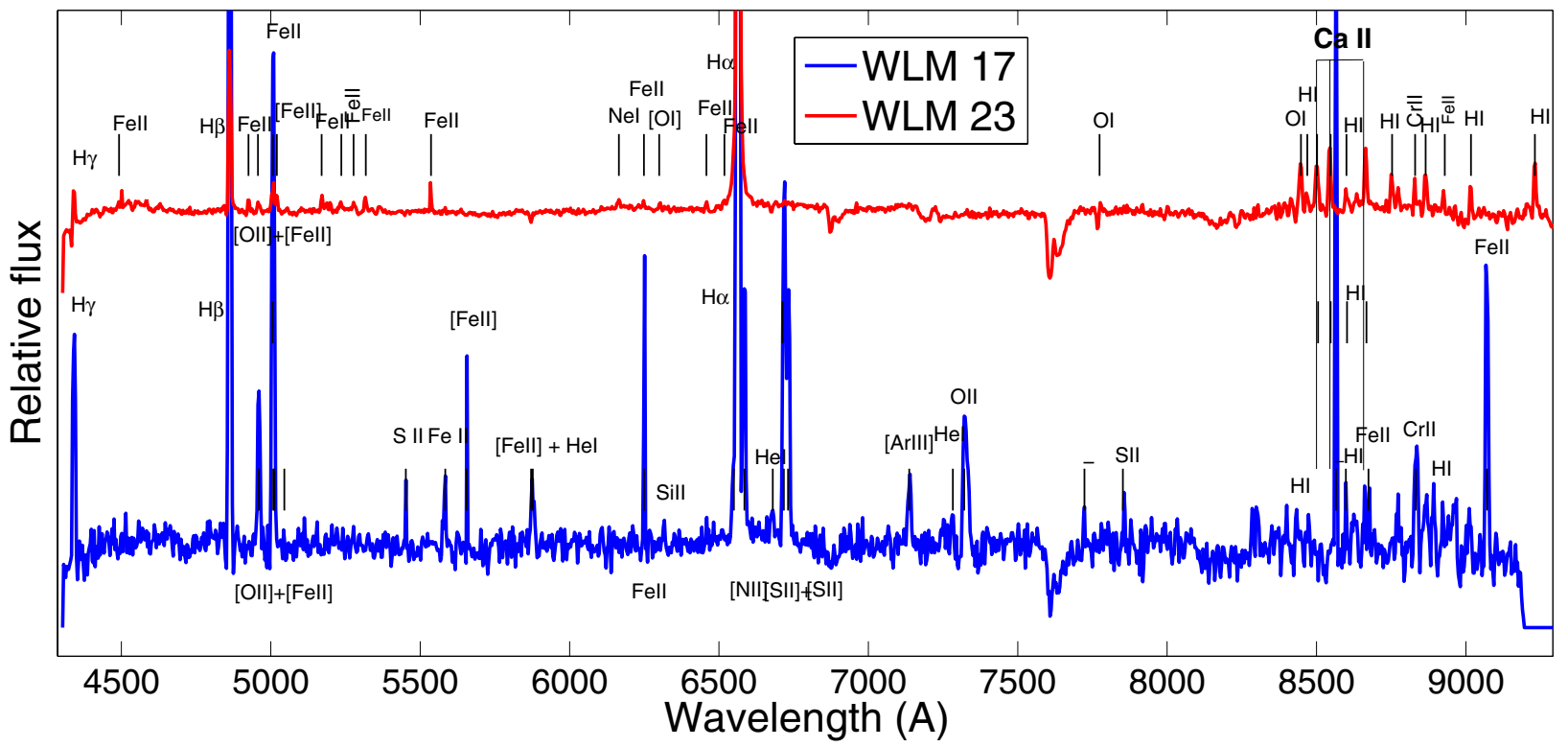

Fig. 7. Normalized spectra of two emission line objects from WLM. Prominent spectral features are labeled.

which we could not identify because the Ca II region is absent from the observed spectra. Moreover, some of the "Unclassified" spectra belong to targets that lie in the red part of the CMDs ([3.6]-[4.5] > $0.2 \mathrm{mag}$ ). These targets could be extreme (dusty) AGB stars, especially the targets whose spectra are very noisy.

Red supergiants are the most numerous class of spectroscopically confirmed dusty massive stars in our sample of dIrr galaxies. We can compare the success of the mid-IR photometric selection criteria with the "classical" optical selection criteria (Massey 1998; Levesque \& Massey 2012). Massey (1998) presented the first attempt to provide a tool with which to distinguish RSG candidates from foreground stars. Using the Kurucz (1992) ATLAS9 stellar atmospheres, the authors showed that it is possible to separate RSGs from foreground giants from a twocolor diagram $(B-V$ vs. $V-R)$. The least-squares model fit, in the formalism of the $B, V$ and $R$ bands, for separating RSGs from foreground contaminants is $B-V=-1.599 \times(V-R)^{2}+4.18 \times$ $(V-R)-1.04+\delta$. The empirical coefficient $\delta$ is added to fit the positions of spectroscopically confirmed RSGs in the program galaxies. Massey (1998), Massey et al. (2009), and Drout et al. (2012) adopted $\delta=0.1$ by fitting the positions of spectroscopically confirmed RSGs in NGC 6822, M31, and M33. All targets that lie above this curve are considered to be candidate RSGs, while targets below the line are considered to be foreground contaminants. The generalization of this dividing curve as a line $B-V=1.25 \times(V-R)+0.45$ has also been used in Levesque \& Massey (2012).

In Fig. 10 we plot all identified RSGs and giants for all program galaxies, including 2 RSGs in IC 1613, 3 in Sextans A from Britavskiy et al. (2014), 11 RSGs in WLM from Levesque \& Massey (2012), and 26 in NGC 6822 from Levesque \& Massey (2012), Patrick et al. (2015), on both the optical $B-V$ vs. $V-R$ two-color diagram and the mid-IR color-magnitude diagram. For targets in NGC 6822 we used Spitzer photometry at [3.6] and [4.5] bands from Khan et al. (2015). Optical colors are taken from Massey et al. (2007c). In the two-color diagram we show both the empirical parabolic curve and the line, discussed above, that separates RSGs from the giants. To compare 


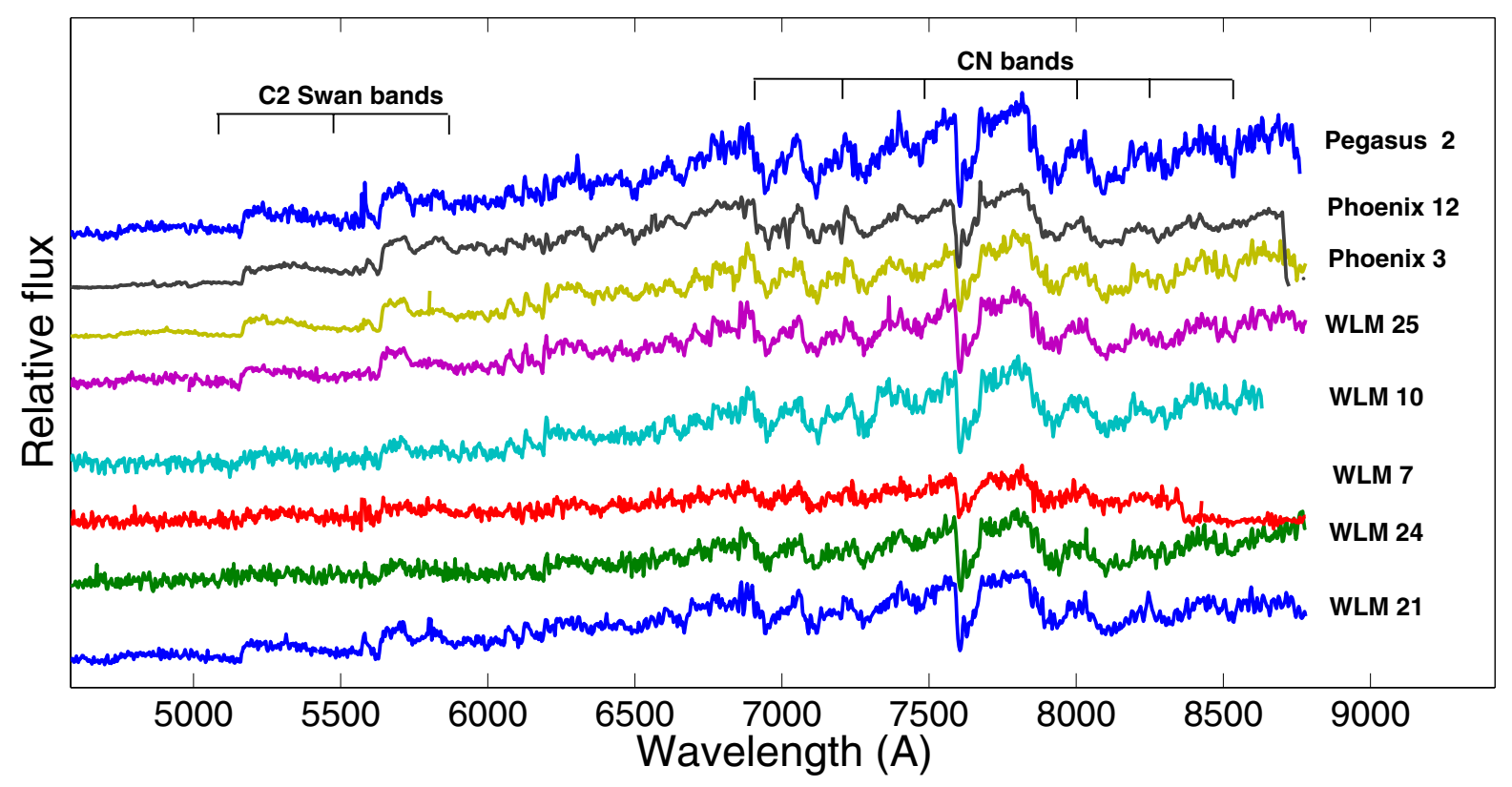

Fig. 8. Spectra of all carbon stars in our sample, with the main molecular features labeled.
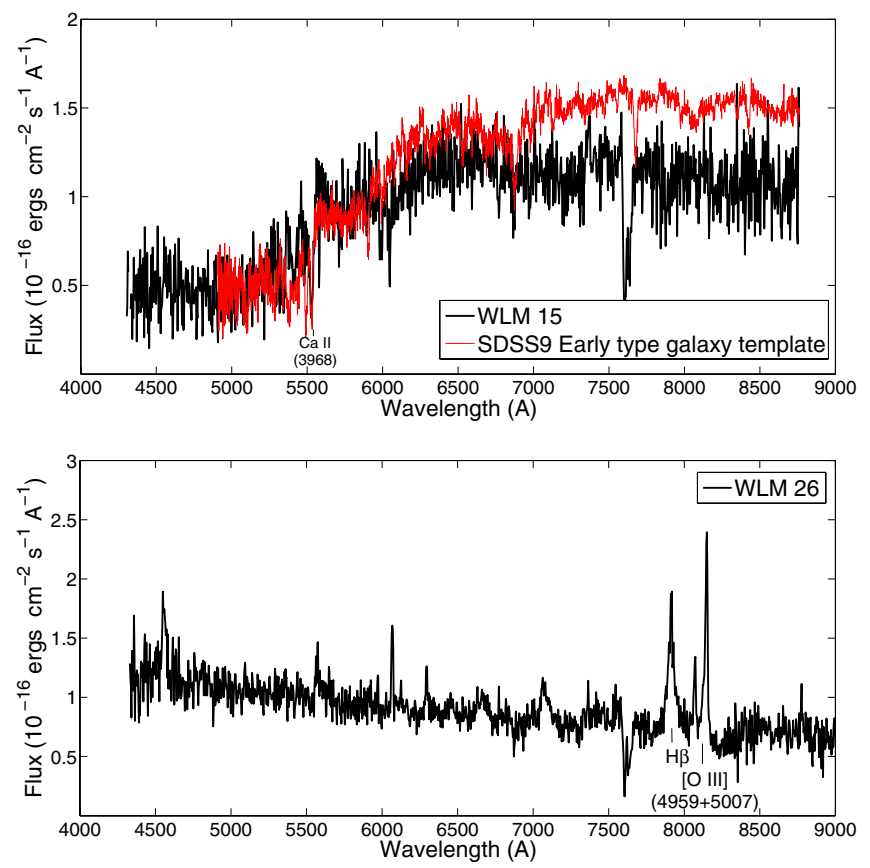

Fig. 9. Spectra of the background galaxy WLM 15 (upper panel) and quasar WLM 26 (lower panel). An SDSS-III early-type galaxy template (red) is superposed on the spectrum of WLM 15 (black).

how the positions of our RSG sample match these cutoff criteria, we calculated the probability density function (PDF) of the position of RSGs on the $B-V$ vs. $V-R$ color magnitude by using a Gaussian kernel density estimation. In Fig. 10 we indicate the contours of the $1 \sigma$ PDF of the location of RSGs and foreground giants in the mid-IR CMD (upper panel) and in the optical two-color diagram (lower panel). The color bar corresponds to the relative value of the probability density that RSGs are located in this specific region. We find that all RSGs are grouped in the narrow region predicted in the two-color diagram, above the empirical cutoff lines. Moreover, the $1 \sigma$ interval of the PDF for the RSG region completely agrees with this parabolic equation. Our analysis indicates that both linear and parabolic cutoff lines (from Massey 1998) can be applied as a tool to separate RSGs from foreground giants. Taking into account that we plotted RSGs from different galaxies with slightly different metallicities, we provide evidence that this dividing line is universal and might be used for all galaxies with precise optical photometry.

The situation is different for the mid-IR selection criteria. The upper panel of Fig. 10 shows that the RSGs have a large dispersion and a high probability of foreground contaminants (as shown by the $1 \sigma$ contour of the PDF of the foreground giants from our sample). These effects make our midIR selection criteria less efficient than the optical colors as a tool for separating RSGs from foreground giants. Furthermore, the cutoff line [3.6]-[4.5] $<0$ that we used for selection does not include all spectroscopically confirmed RSGs. To generalize this statement, we plotted all spectroscopically confirmed RSGs in the SMC: 59 from Bonanos et al. (2010), 83 from González-Fernández et al. (2015), and the LMC: 96 from Bonanos et al. (2009), 96 from González-Fernández et al. (2015) together with RSGs from our sample of dIrr galaxies in Fig. 11. We plot the WISE W1 $(3.353 \mu \mathrm{m})$ and W2 $(4.603 \mu \mathrm{m})$ photometry of the RSGs from González-Fernández et al. (2015), which are very close to the Spitzer [3.6] and [4.5] bands. We find the majority of new RSGs in the SMC and the LMC to be fainter compared with the previously known RSGs. The difference in absolute magnitude most likely arises because the WISE photometry has a higher sensitivity, even though the resolution is poorer than the Spitzer photometry for the Magellanic Clouds. There is also a difference in the [3.6]-[4.5] colors for the RSGs in the LMC vs. SMC. The maximum (peak) of the PDF for RSGs from the LMC has a bluer color ([3.6]-[4.5] $=-0.17$ mag) and brighter magnitude $\left(M_{[3.6]}=-10.15 \mathrm{mag}\right)$ than the position of the maximum PDF for the RSGs from the SMC ([3.6]-[4.5] $=-0.02 \mathrm{mag}, M_{[3.6]}=-9.51 \mathrm{mag}$ ). This difference originates in the different metallicities of the LMC and the SMC, which affect the depth of CO bands at [4.5] $\mu \mathrm{m}$, and as a result the [3.6]-[4.5] color. 
N. E. Britavskiy et al.: Identification of dusty massive stars in nearby galaxies

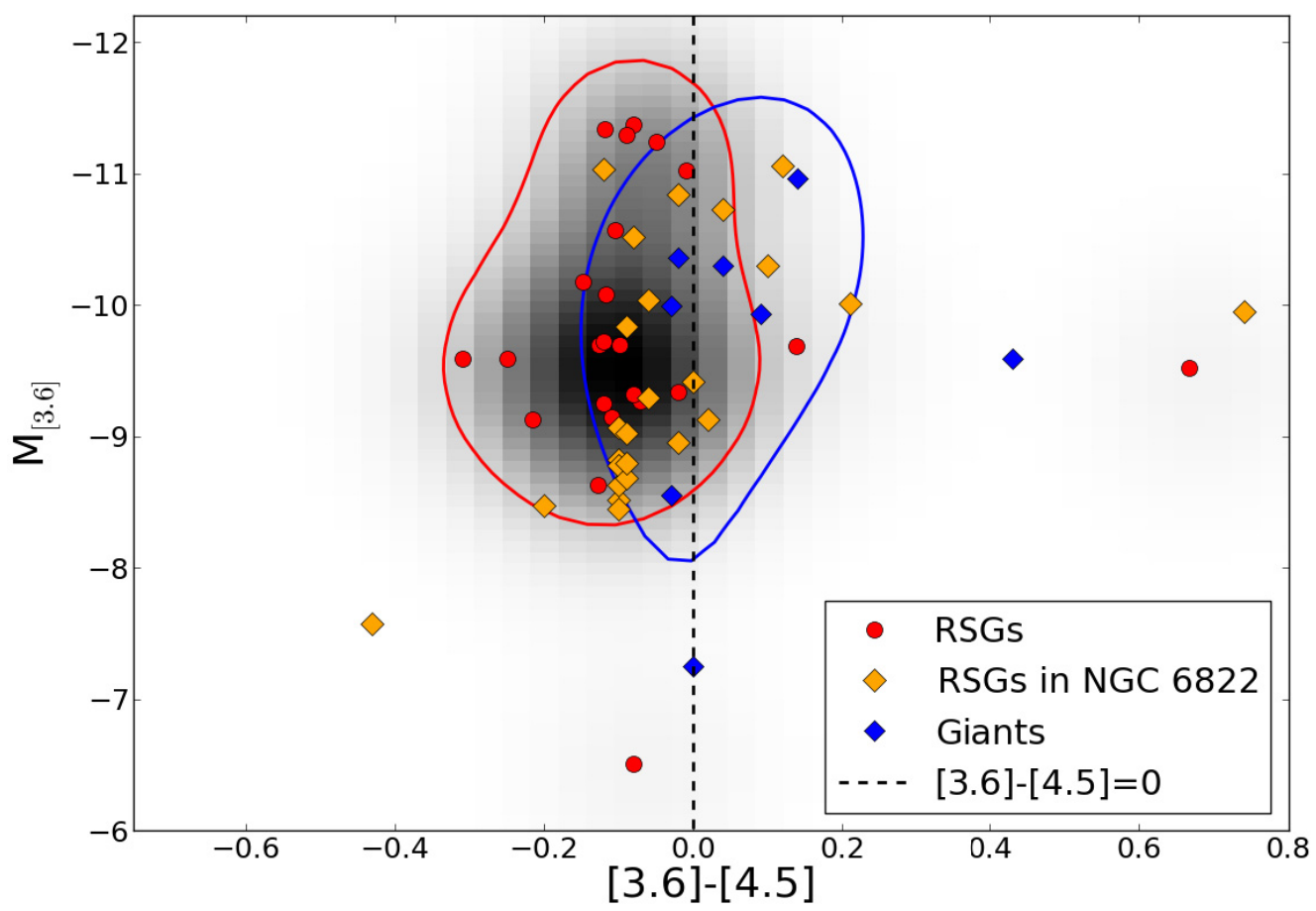

0.90

0.60

0.45

0.30

0.15

0.00

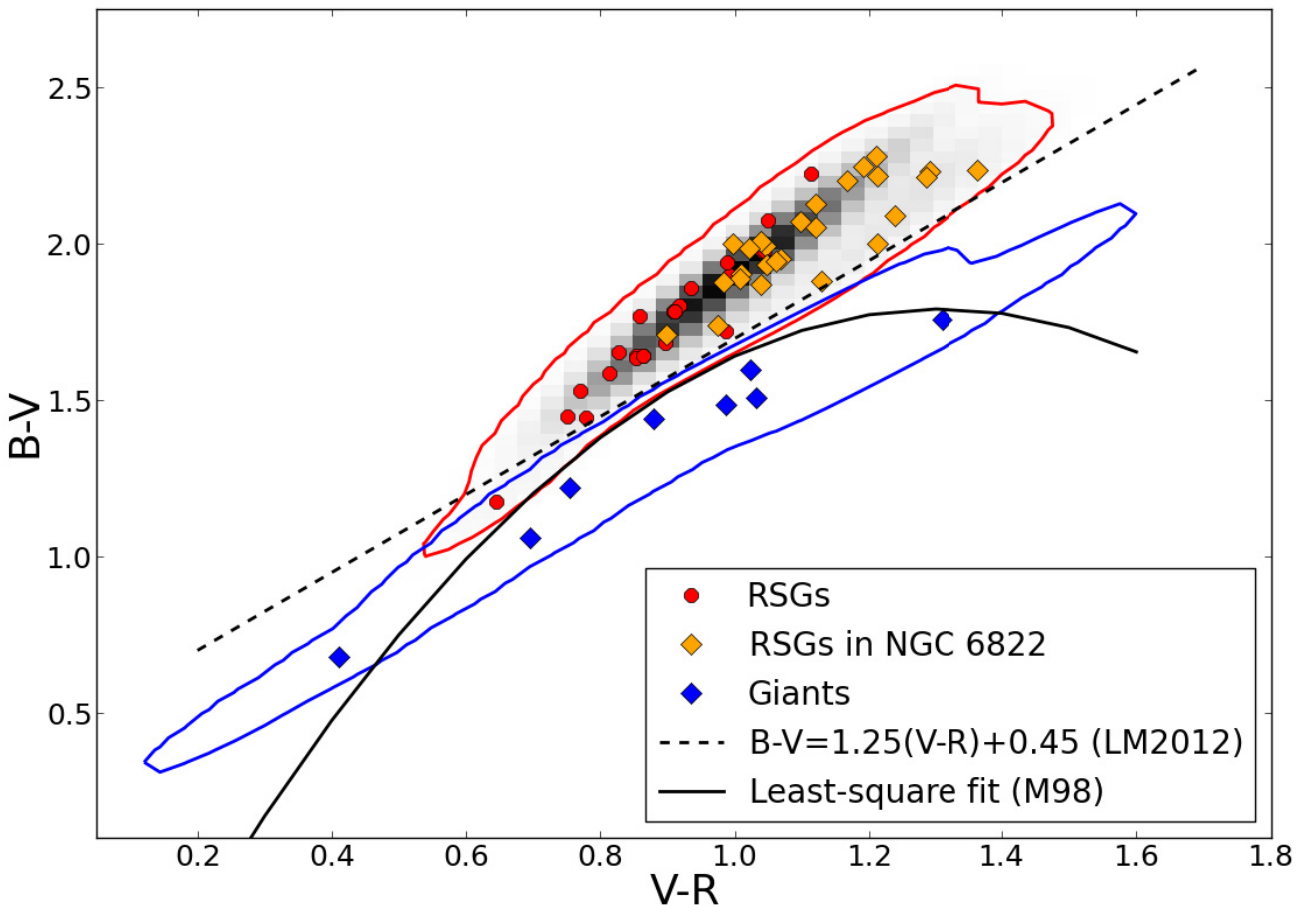

Fig. 10. $M_{[3.6]}$ vs. [3.6]-[4.5] CMD (upper panel) and two-color optical diagram (lower panel) for all spectroscopically confirmed RSGs and giants in our four program galaxies, including known RSGs from the literature in WLM, NGC 6822, IC 1613, and Sextans A. RSGs in NGC 6822 are marked separately because of the different source mid-IR photometry. RSGs are indicated by red filled circles, giants by blue filled diamonds, and the color contours correspond to a $1 \sigma$ dispersion of the Gaussian PDF. The probability density function for the whole sample of RSGs is shown in gray; the color bar corresponds to the relative value of the PDF. The dashed line at [3.6]-[4.5] = 0 demarcates our mid-IR selection criteria for identifying RSGs. The dashed line in the two-color diagram corresponds to the empirical dividing line that separates RSGs from foreground giant candidates defined in Levesque \& Massey (2012). The solid line corresponds to a least-squares fit model of separating RSG candidates from foreground candidates, provided in Massey (1998).

We added the same sample of RSGs from the Magellanic Clouds to the optical two-color diagram (lower panel of Fig. 11). Optical colors for this sample of RSGs are taken from Massey (2002). For such a large sample of RSGs the large scatter of their positions in the two-color diagram is evident, which is mainly due to the accuracy of the optical photometry. Nevertheless, the majority $(\approx 70 \%)$ of the spectroscopically confirmed RSGs satisfies the optical selection criteria. Comparing this success rate in the optical selection criteria with the number of RSGs that satisfy the mid-IR selection criteria $(\approx 90 \%)$, we can conclude 

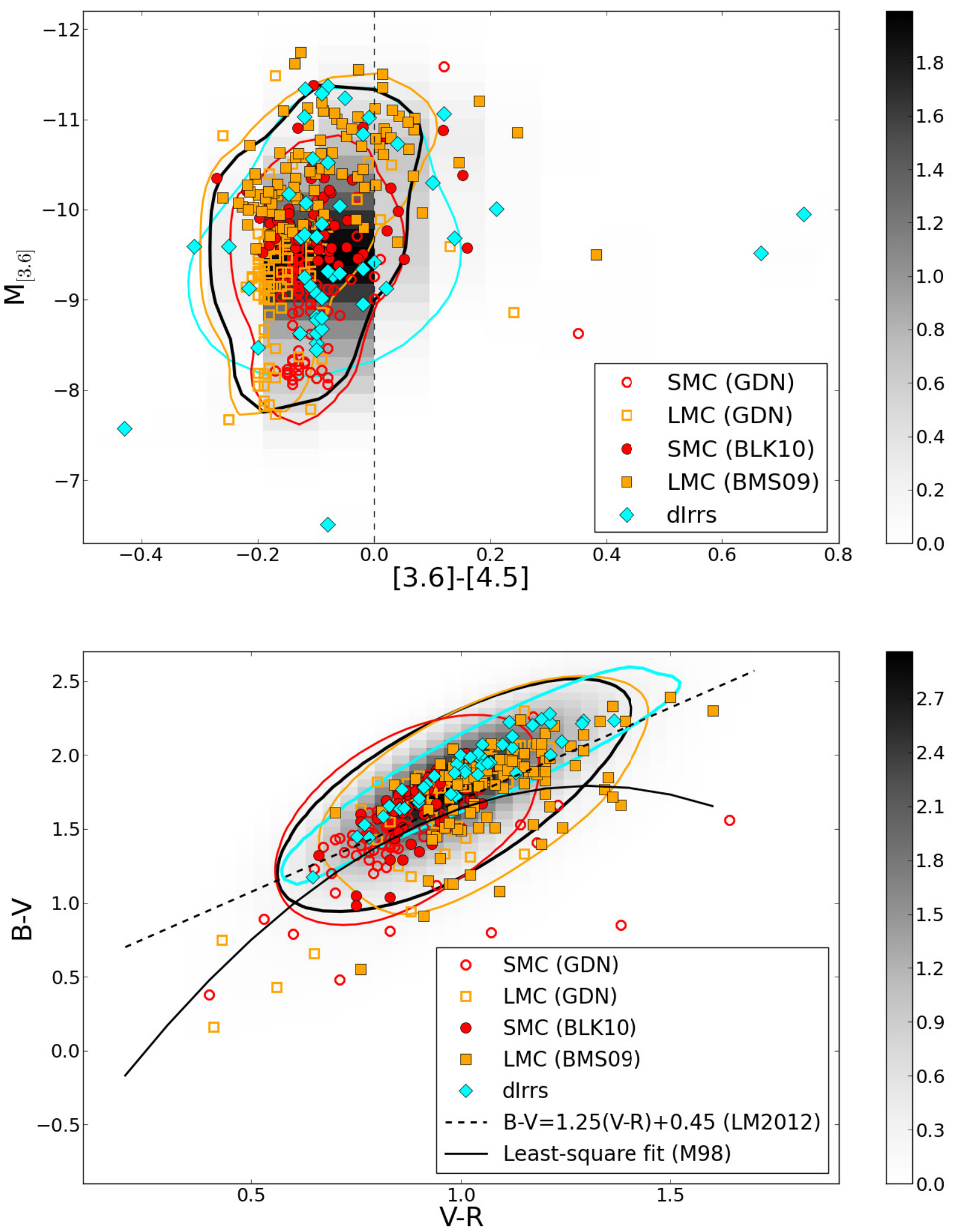

Fig. 11. $M_{[3.6]}$ vs. [3.6]-[4.5] CMD (upper panel) and $B-V$ vs. $V-R$ diagram (lower panel) for spectroscopically confirmed RSGs in six Local Group dIrrs (Phoenix, Pegasus, Sextans A, WLM, IC 1613, and NGC 6822), the LMC, and the SMC. The RSGs from González-Fernández et al. (2015) are labeled "GDN"; RSGs labeled "BLK10" and "BMS09" are taken from Bonanos et al. (2010, 2009) respectively. The color contours correspond to a $1 \sigma$ dispersion of the PDF for all RSGs for each galaxy, respectively. The black contour corresponds to a $1 \sigma$ dispersion for the PDF of all RSGs in listed galaxies. The probability density function for all RSGs is highlighted in gray, the color bar corresponds to relative values of the PDF. The dashed line at [3.6]-[4.5] = 0 demarcates our selection criteria for identifying RSGs. The dashed line and the solid line in the two-color diagram have the same meaning as in Fig. 10. Optical photometry for RSGs in the Magellanic Clouds is adopted from Massey (2002). 
Table 3. Summary classification of our 79 observed targets.

\begin{tabular}{lllllllll}
\hline \hline ID & $\begin{array}{l}\text { All observed } \\
\text { targets }\end{array}$ & Unclassified & $\begin{array}{l}\text { Spectral type } \\
\text { only }\end{array}$ & Giants & RSGs & $\begin{array}{l}\text { Em. line } \\
\text { objects }\end{array}$ & $\begin{array}{l}\text { Background } \\
\text { objects }\end{array}$ & Carbon stars \\
\hline Pegasus & $\mathbf{1 1}(+8)$ & $\mathbf{2}(+7)$ & $\mathbf{3}$ & $\mathbf{4}$ & $\mathbf{2}$ & $\mathbf{0}$ & $\mathbf{0}$ & $(+1)$ \\
Phoenix & $\mathbf{2}(+12)$ & $\mathbf{0}(+5)$ & $\mathbf{2}(+2)$ & $\mathbf{0}(+2)$ & $\mathbf{0}(+1)$ & $\mathbf{0}$ & $\mathbf{0}$ & $(+2)$ \\
Sextans A & $\mathbf{1 5}$ & $\mathbf{5}$ & $\mathbf{2}$ & $\mathbf{1}$ & $\mathbf{7}$ & $\mathbf{0}$ & $\mathbf{0}$ & $(0)$ \\
WLM & $\mathbf{1 5}(+16)$ & $\mathbf{5}(+8)$ & $\mathbf{3}(+1)$ & $\mathbf{0}(+1)$ & $\mathbf{4}$ & $\mathbf{2}$ & $\mathbf{1}(+1)$ & $(+5)$ \\
\hline Total & $\mathbf{4 3}(+36)$ & $\mathbf{1 2}(+20)$ & $\mathbf{1 0}(+3)$ & $\mathbf{5}(+3)$ & $\mathbf{1 3}(+1)$ & $\mathbf{2}$ & $\mathbf{1}(+1)$ & $(+8)$ \\
$\%$ & $\mathbf{1 0 0}$ & $\mathbf{2 8}$ & $\mathbf{2 3}$ & $\mathbf{1 2}$ & $\mathbf{3 0}$ & $\mathbf{5}$ & $\mathbf{2}$ & - \\
\hline
\end{tabular}

Notes. The number of targets that satisfy our selection criteria are indicated by bold font. Targets that were randomly selected to fill the MXU slits are given in parentheses.

that the mid-IR selection criteria are useful for selecting dusty massive stars, even though these criteria are not so efficient for separating them from foreground contaminants, in contrast with the optical selection criteria (as shown in Fig. 10).

We conclude that RSGs group in a broader region in the mid-IR CMD, in contrast to the narrow region defined on the optical two-color diagram, which makes optical selection criteria more reliable when high-quality optical photometry exists for the investigated galaxies. However, the mid-IR criteria are useful for the large number of galaxies with Spitzer imaging that lack deep optical photometry. This work together with Britavskiy et al. (2014) increased the sample of spectroscopically confirmed RSGs in dIrr galaxies in the Local Group by 12 (27\%): we have identified 2 RSGs in IC 1613, 7 RSGs in Sextans A, 1 RSG in Phoenix, 2 RSGs in Pegasus. Before these works, there were 44 RSGs spectroscopically confirmed in dIrrs of the Local Group: 33 RSGs were known in NGC 6822 (Massey 1998; Levesque \& Massey 2012; Patrick et al. 2015), and 11 RSGs were known in WLM (Levesque \& Massey 2012).

\section{Summary}

We here presented an attempt to complete the census of dusty massive stars in a sample of four dwarf irregular galaxies in the Local Group: Pegasus, Phoenix, Sextans A, and WLM. We used mid-IR selection criteria based on the [3.6] and [4.5] bands for identifying dusty types of massive stars such as RSGs, LBVs, and $\mathrm{sgB}[\mathrm{e}]$ stars. We performed a spectroscopic analysis for 79 sources. As a result of our work, 13 RSGs were identified ( 6 of them are newly discovered) together with 2 emission line objects (one of them is a newly identified iron star) and 1 candidate yellow supergiant. The large sample of spectroscopically defined RSGs enabled us to investigate and revise the mid-IR and optical selection criteria. We provided evidence that the most precise method for selecting RSGs from foreground objects is an optical $B-V$ vs. $V-R$ color-color diagram with the linear and the parabolic separation line of Massey (1998). The mid-IR selection criteria are less efficient, but with available Spitzer mid-IR photometry (Boyer et al. 2015b) for 13 dwarf irregular galaxies in the Local Group, our tool provides an opportunity to select candidate dusty massive stars in star-forming dIrrs in cases where accurate optical photometry does not exist or is not deep enough. The mid-IR selection criteria can be applied both to galaxies in the Local Group and to more distant galaxies (e.g., M83; Williams et al. 2015) by using midinfrared photometry from other surveys, for instance, from the Local Volume Legacy (Dale et al. 2009) and the SIRTF Nearby Galaxy Survey (Kennicutt et al. 2003). At this stage, we cannot verify selection criteria of emission line objects because only a small number of these stars were identified. This work provides a basis for future identification of dusty massive stars in the Local Group. Moreover, newly discovered RSGs can be used to investigate metallicities and the properties of their host galaxies (e.g., Davies et al. 2013; Patrick et al. 2015).

Acknowledgements. We thank the anonymous referee for helpful comments that have improved the manuscript. N. Britavskiy and A.Z. Bonanos acknowledge funding by the European Union (European Social Fund) and National Resources under the "ARISTEIA" action of the Operational Programme "Education and Lifelong Learning" in Greece. We would like to thank A. Miroshnichenko for useful discussions on the identification of emission lines in spectra. This research has made use of NASA's Astrophysics Data System Bibliographic Services and the VizieR catalogue access tool, CDS, Strasbourg, France. Funding for SDSS-III has been provided by the Alfred P. Sloan Foundation, the Participating Institutions, the National Science Foundation, and the US Department of Energy Office of Science.

\section{References}

Ahn, C. P., Alexandroff, R., Allende Prieto, C., et al. 2012, ApJS, 203, 21 Bagnulo, S., Jehin, E., Ledoux, C., et al. 2003, The Messenger, 114, 10 Battinelli, P., \& Demers, S. 2000, AJ, 120, 1801

Battinelli, P., \& Demers, S. 2004, A\&A, 416, 111

Bonanos, A. Z., Massa, D. L., Sewilo, M., et al. 2009, AJ, 138, 1003 Bonanos, A. Z., Lennon, D. J., Köhlinger, F., et al. 2010, AJ, 140, 416

Boyer, M. L., Skillman, E. D., van Loon, J. T., Gehrz, R. D., \& Woodward, C. E. 2009, ApJ, 697, 1993

Boyer, M. L., McQuinn, K. B. W., Barmby, P., et al. 2015a, ApJ, 800, 51

Boyer, M. L., McQuinn, K. B. W., Barmby, P., et al. 2015b, ApJS, 216, 10

Britavskiy, N. E., Bonanos, A. Z., Mehner, A., et al. 2014, A\&A, 562, A75

Cardiel, N. 2010, Astrophysics Source Code Library

[record ascl: 1010.046]

Cenarro, A. J., Cardiel, N., Gorgas, J., et al. 2001, MNRAS, 326, 959

Cenarro, A. J., Gorgas, J., Cardiel, N., Vazdekis, A., \& Peletier, R. F. 2002, MNRAS, 329, 863

Chentsov, E. L., Ermakov, S. V., Klochkova, V. G., et al. 2003, A\&A, 397, 1035

Clark, J. S., Castro, N., Garcia, M., et al. 2012, A\&A, 541, A146

Dale, D. A., Cohen, S. A., Johnson, L. C., et al. 2009, ApJ, 703, 517

Davies, B., Kudritzki, R.-P., Plez, B., et al. 2013, ApJ, 767, 3

Drout, M. R., Massey, P., \& Meynet, G. 2012, ApJ, 750, 97

Freudling, W., Romaniello, M., Bramich, D. M., et al. 2013, A\&A, 559, A96

Gallart, C., Martínez-Delgado, D., Gómez-Flechoso, M. A., \& Mateo, M. 2001, AJ, 121, 2572

Ginestet, N., Carquillat, J. M., Jaschek, M., \& Jaschek, C. 1994, A\&AS, 108, 359

González-Fernández, C., Dorda, R., Negueruela, I., \& Marco, A. 2015, A\&A, 578, A3

Hodge, P., \& Miller, B. W. 1995, ApJ, 451, 176

Hodge, P. W., Dolphin, A. E., Smith, T. R., \& Mateo, M. 1999, ApJ, 521, 577

Humphreys, R. M., Weis, K., Davidson, K., Bomans, D. J., \& Burggraf, B. 2014, ApJ, 790, 48

Keenan, P. C. 1993, PASP, 105, 905

Kennicutt, Jr., R. C., Armus, L., Bendo, G., et al. 2003, PASP, 115, 928

Khan, R., Stanek, K. Z., Prieto, J. L., et al. 2010, ApJ, 715, 1094

Khan, R., Stanek, K. Z., Kochanek, C. S., \& Bonanos, A. Z. 2011, ApJ, 732, 43

Khan, R., Stanek, K. Z., \& Kochanek, C. S. 2013, ApJ, 767, 52 
Khan, R., Stanek, K. Z., Kochanek, C. S., \& Sonneborn, G. 2015, ApJS, 219, 42 Kondratiev, V., Lorimer, D., McLaughlin, M., \& Ransom, S. 2013, in IAU Symp. 291, ed. J. van Leeuwen, 431

Kozłowski, S., Onken, C. A., Kochanek, C. S., et al. 2013, ApJ, 775, 92

Kraus, M., Cidale, L. S., Arias, M. L., Oksala, M. E., \& Borges Fernandes, M. 2014, ApJ, 780, L10

Kurucz, R. L. 1992, in The Stellar Populations of Galaxies, eds. B. Barbuy, \& A. Renzini, IAU Symp. 149, 225

Levesque, E. M. 2010, New Astron. Rev., 54, 1

Levesque, E. M. 2013, in EAS Pub. Ser., 60, eds. P. Kervella, T. Le Bertre, \& G. Perrin, 269

Levesque, E. M., \& Massey, P. 2012, AJ, 144, 2

Martínez-Delgado, D., Gallart, C., \& Aparicio, A. 1999, AJ, 118, 862

Massey, P. 1998, ApJ, 501, 153

Massey, P. 2002, ApJS, 141, 81

Massey, P., Olsen, K. A. G., Hodge, P. W., et al. 2006, AJ, 131, 2478

Massey, P., Levesque, E. M., Olsen, K. A. G., Plez, B., \& Skiff, B. A. 2007a, ApJ, 660, 301
Massey, P., McNeill, R. T., Olsen, K. A. G., et al. 2007b, AJ, 134, 2474 Massey, P., Olsen, K. A. G., Hodge, P. W., et al. 2007c, AJ, 133, 2393 Massey, P., Silva, D. R., Levesque, E. M., et al. 2009, ApJ, 703, 420 Mateo, M. L. 1998, ARA\&A, 36, 435

McConnachie, A. W. 2012, AJ, 144, 4

Menzies, J., Feast, M., Whitelock, P., et al. 2008, MNRAS, 385, 1045

Negueruela, I., Marco, A., González-Fernández, C., et al. 2012, A\&A, 547, A15

Neugent, K. F., \& Massey, P. 2011, ApJ, 733, 123

Patrick, L. R., Evans, C. J., Davies, B., et al. 2015, ApJ, 803, 14

Rayner, J. T., Cushing, M. C., \& Vacca, W. D. 2009, ApJS, 185, 289

Smith, N. 2014, ARA\&A, 52, 487

Tolstoy, E. 1999, in The Stellar Content of Local Group Galaxies, eds. P. Whitelock, \& R. Cannon, IAU Symp., 192, 218

Verhoelst, T., van der Zypen, N., Hony, S., et al. 2009, A\&A, 498, 127

Williams, S. J., Bonanos, A. Z., Whitmore, B. C., Prieto, J. L., \& Blair, W. P. 2015, A\&A, 578, A100

Zethson, T., Johansson, S., Hartman, H., \& Gull, T. R. 2012, A\&A, 540, A133

Pages 15 to 17 are available in the electronic edition of the journal at http://www . aanda. org 
N. E. Britavskiy et al.: Identification of dusty massive stars in nearby galaxies

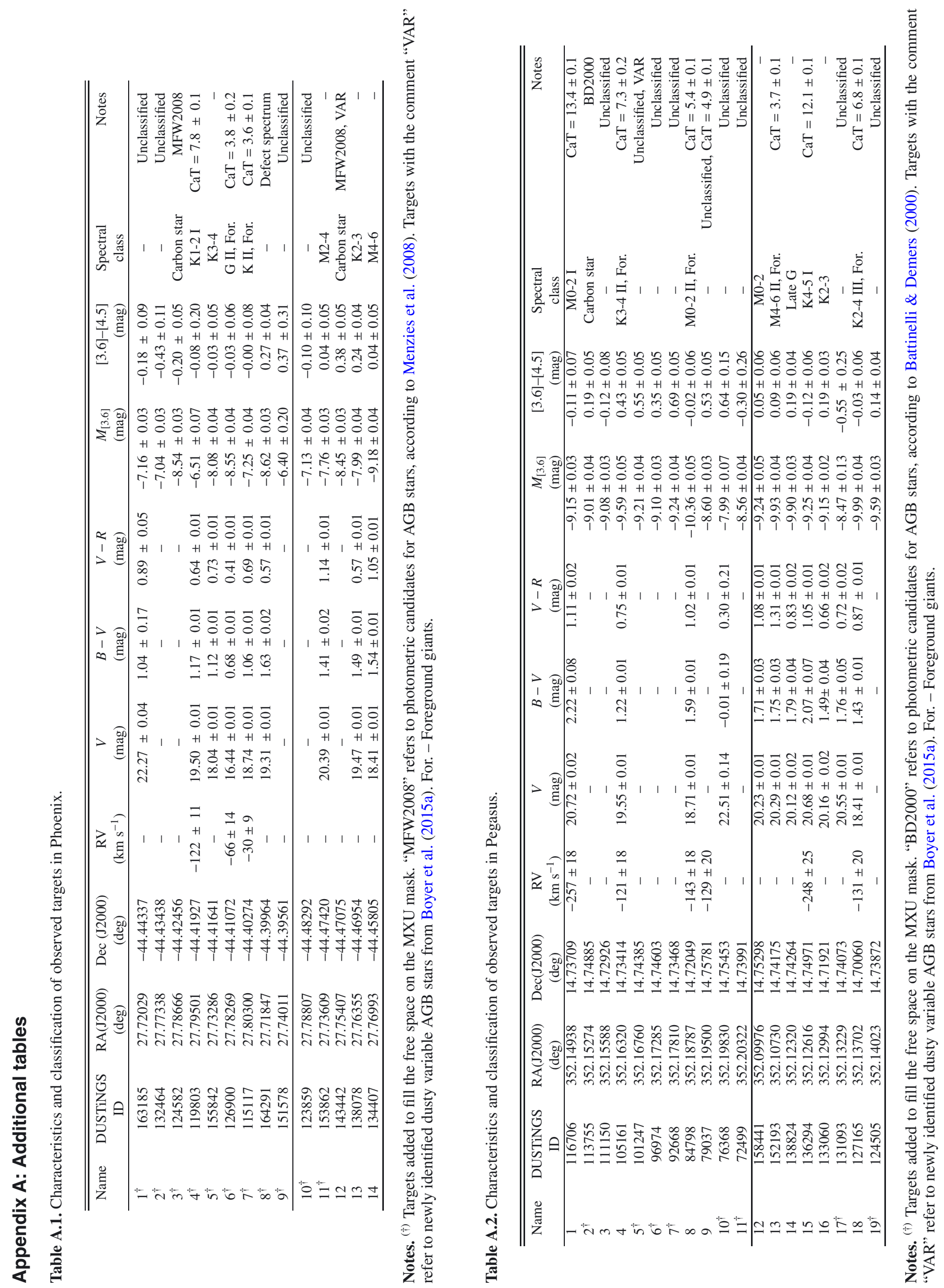


A\&A 584, A33 (2015)

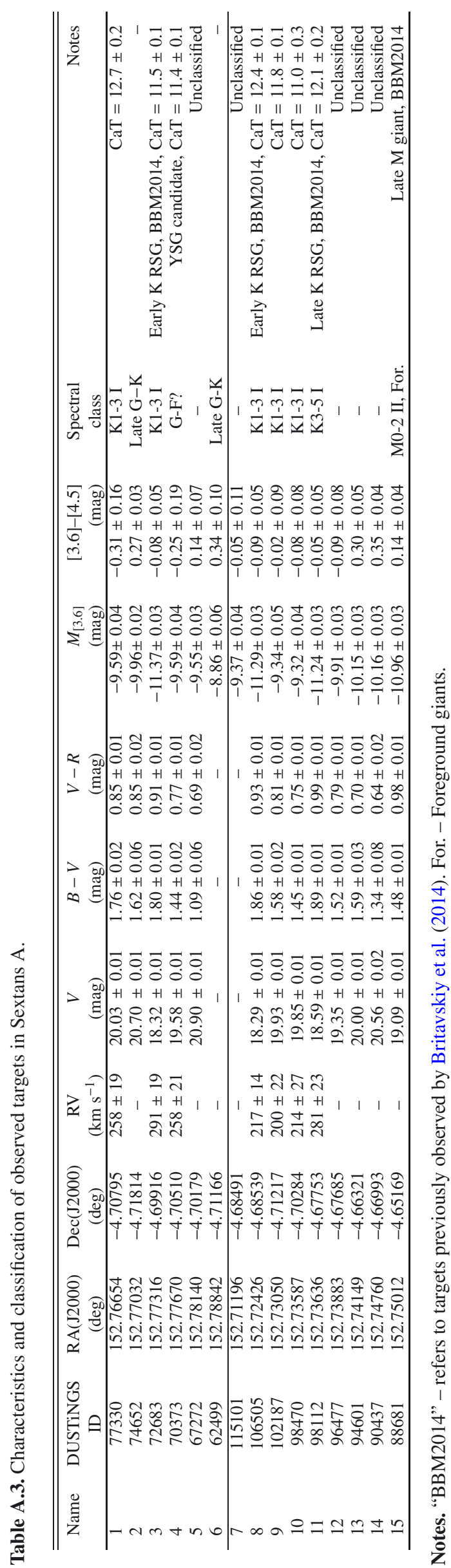


N. E. Britavskiy et al.: Identification of dusty massive stars in nearby galaxies

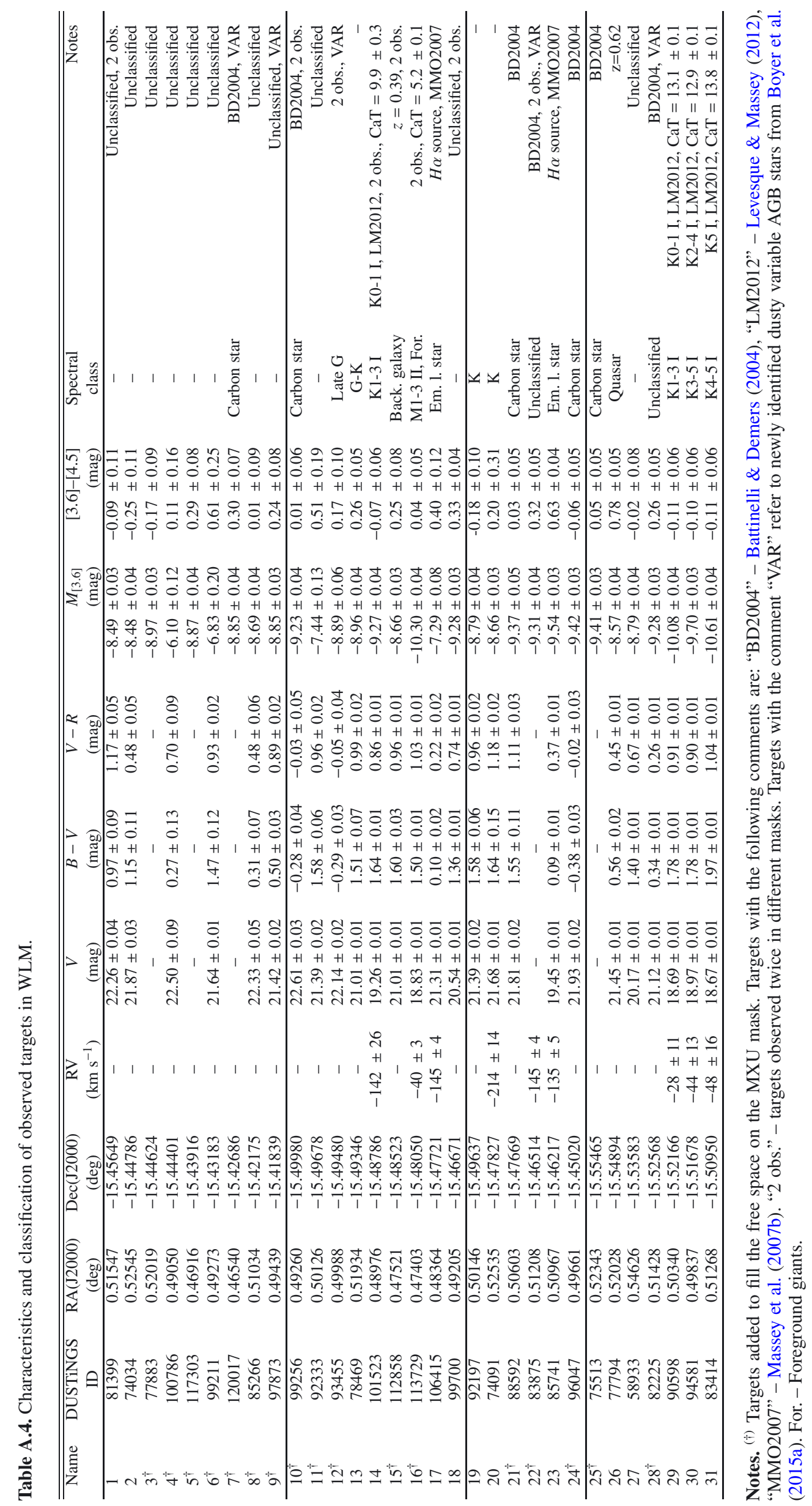

